\title{
A!
}

This is an electronic reprint of the original article.

This reprint may differ from the original in pagination and typographic detail.

Virtanen, P.; Ronzani, A.; Giazotto, F.

\section{Josephson Photodetectors via Temperature-to-Phase Conversion}

Published in:

Physical Review Applied

DOI:

10.1103/PhysRevApplied.9.054027

Published: 17/05/2018

Document Version

Publisher's PDF, also known as Version of record

Please cite the original version:

Virtanen, P., Ronzani, A., \& Giazotto, F. (2018). Josephson Photodetectors via Temperature-to-Phase

Conversion. Physical Review Applied, 9(5), 1-15. [054027]. https://doi.org/10.1103/PhysRevApplied.9.054027

This material is protected by copyright and other intellectual property rights, and duplication or sale of all or part of any of the repository collections is not permitted, except that material may be duplicated by you for your research use or educational purposes in electronic or print form. You must obtain permission for any other use. Electronic or print copies may not be offered, whether for sale or otherwise to anyone who is not an authorised user. 


\title{
Josephson Photodetectors via Temperature-to-Phase Conversion
}

\author{
P. Virtanen, ${ }^{1, *}$ A. Ronzani ${ }^{2}$ and F. Giazotto ${ }^{1, \dagger}$ \\ ${ }^{1}$ NEST, Instituto Nanoscienze-CNR and Scuola Normale Superiore, I-56127 Pisa, Italy \\ ${ }^{2}$ Low Temperature Laboratory, Department of Applied Physics, Aalto University, 00076 Aalto, Finland
}

(Received 5 February 2018; published 17 May 2018)

\begin{abstract}
We theoretically investigate the temperature-to-phase conversion (TPC) process occurring in $\mathrm{dc}$ superconducting quantum interferometers based on superconductor-normal-metal-superconductor $(S-N-S)$ mesoscopic Josephson junctions. In particular, we predict the temperature-driven rearrangement of the phase gradients in the interferometer under the fixed constraints of fluxoid quantization and supercurrent conservation. This mechanism allows sizeable phase variations across the junctions for suitable structure parameters and temperatures. We show that the TPC can be a basis for sensitive singlephoton sensors or bolometers. We propose a radiation detector realizable with conventional materials and state-of-the-art nanofabrication techniques. Integrated with a superconducting quantum-interference proximity transistor as a readout setup, an aluminum-based TPC calorimeter can provide a large signal-to-noise ratio $>100$ in the $10-\mathrm{GHz}-10-\mathrm{THz}$ frequency range and a resolving power larger than $10^{2}$ below $50 \mathrm{mK}$ for terahertz photons. In the bolometric operation, electrical noise equivalent power of approximately $10^{-22} \mathrm{~W} / \sqrt{\mathrm{Hz}}$ is predicted at $50 \mathrm{mK}$. This device can be attractive as a cryogenic singlephoton sensor operating in the giga- and terahertz regime with applications in dark-matter searches.
\end{abstract}

DOI: $10.1103 /$ PhysRevApplied.9.054027

\section{INTRODUCTION}

Detecting radiation in the microwave to terahertz regime has a range of applications from astrophysics to quantum devices. For applications where cooling to low temperatures is available, superconducting sensors provide a way to construct high-sensitivity detectors, both for bolometric detection of continuous power [1-4] and also toward the detection of few photons [5-9].

Thermal superconducting radiation detectors $[1-3,10]$ generally make use of the sensitive dependence of superconducting electrical response on the local electronic temperature, which changes as photons are absorbed. The specific way this response is utilized varies in different detectors. Kinetic inductance $[1,11,12]$ and Josephson superconductor-normal-metal-superconductor- $\quad(S-N-S)$ [8,13-15] based detectors make use of the temperature dependence of the supercurrent. The changes in the current response can be detected, e.g., via resonant circuits [11] or inductively or directly $[1,16]$ coupled superconducting quantum-interference-device (SQUID) sensors. In the direct coupling scheme, variation of the critical current or inductance in the detector embedded as one junction of a dc SQUID affects the distribution of phase differences across the second junction and the current balance, which can be detected. Similarly, if the two junctions reside in a

\footnotetext{
*pauli.virtanen@nano.cnr.it
}

francesco.giazotto@sns.it closed superconducting ring, the result is temperature-tophase difference conversion (TPC), which is the basis of the device discussed below.

The phase difference across a superconducting junction can be generally determined via the current flowing through it. However, in $S-N-S$ and weak links, it is also possible to determine the value via tunnel-junction spectroscopy-this forms the basis for the superconducting quantum-interference proximity transistor (SQUIPT) [17]. In a SQUIPT, the phase difference is measured by observing the tunneling current through a tunnel junction [3] connected to the middle of the weak link. Such measurements can also be made at high bandwidth $[18,19]$ enabling fast measurement timescales that are required for calorimetry.

Superconducting weak links are useful in hot-electron bolometers (HEBs) in that the superconductivity inhibits electronic heat conduction out of the detector region $[1,2,20]$, improving sensitivity via reduction of the intrinsic thermal-fluctuation noise. For bolometric devices, another key quantity is the detector heat capacity $[1,2]$, reducing which lowers the minimum detectable energy and reduces the thermal response time. These parameters can be adjusted to optimize the detector performance within the constraints of the readout method.

In this paper, we propose a mesoscopic superconducting bolometer and calorimeter for single-photon and continuous power detection in the gigahertz to terahertz frequency range. The operation is based on temperature-dependent 


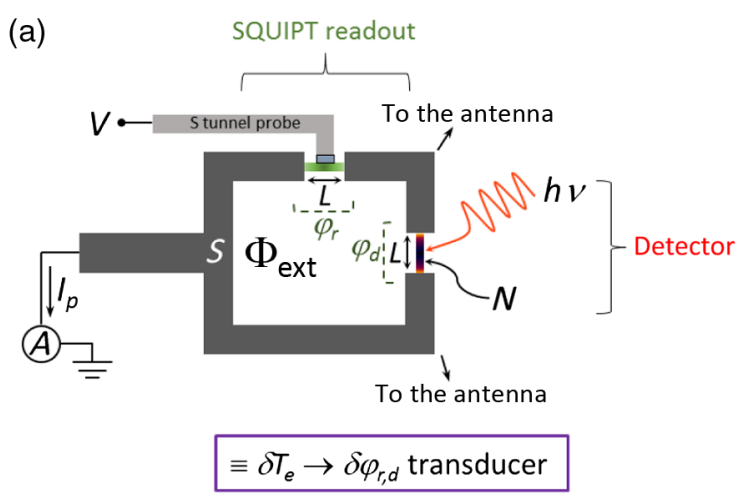

(b)

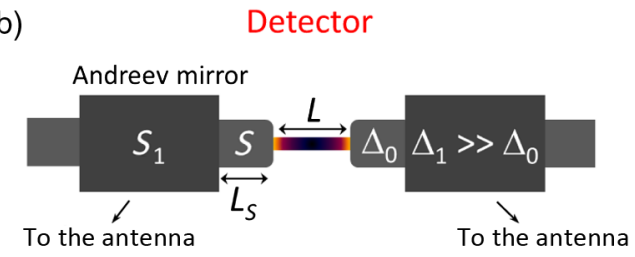

FIG. 1. (a) Scheme of a dc superconducting quantum interferometer realized with two $S-N-S$ Josephson junctions. Absorption of electromagnetic radiation of energy $h \nu$ increases the electronic temperature $\left(T_{e}\right)$ in the $N$ region of the detector junction thereby suppressing the Josephson supercurrent circulating in the loop. The suppression leads to a variation of phase difference $\left(\varphi_{d, r}\right)$ across both Josephson junctions and to a modification of the DOS in their $N$ regions. The DOS in the readout junction is probed via a SQUIPT used as the readout circuit. The structure, therefore, operates as a temperature-to-phase converter, i.e., $\delta T_{e} \rightarrow \delta \varphi_{d, r} . L$ denotes the length of the junctions, and $\boldsymbol{\Phi}_{\text {ext }}$ is the applied magnetic flux. (b) Enlargement of the detector in the vicinity of the weak-link region: Superconducting electrodes $S_{1}$ with energy gap $\Delta_{1} \gg \Delta_{0}\left(\Delta_{0}\right.$ is the zero-temperature energy gap in $\left.S\right)$ in good electric contact with the loop constitute Andreev mirrors for the energy absorbed by quasiparticles in the $N$ region. The Andreev reflection allows energy relaxation in the detector junction to occur predominantly via electron-phonon interaction with lattice phonons in the enclosed $N$ and $S$ regions (see text). $L_{S}$ is the length of the $S$ portions in direct contact with the $N$ nanowire.

kinetic inductance change of a superconducting weak link resulting in TPC in a superconducting ring and its nonlocal readout via an integrated SQUIPT sensor (see Fig. 1). The measurement scheme can accommodate a small detector volume, enabling small heat conductivity and heat capacity, which results in reduced intrinsic thermal noise and low minimum detectable energy and response time. In the weak-link device, these quantities are also tunable via the magnetic flux. For the readout scheme, we predict temperature sensitivities of tens of $\mathrm{nK} / \sqrt{\mathrm{Hz}}$ in a temperature range tunable over $T=10 \mathrm{mK}$ to $1 \mathrm{~K}$ with the choice of the magnetic flux. We provide an appropriate simplified theoretical model for the operation and thermal response and analyze the main performance characteristics in the bolometric and calorimetric modes. In calorimetric operation, we predict signal-to-noise ratios up to 100 in the
$10 \mathrm{GHz}$ to $10 \mathrm{THz}$ range and a resolving power larger than 100 for single photons at detector bath temperatures of $50 \mathrm{mK}$, with characteristic thermal time $\tau \sim 10-500 \mu \mathrm{s}$. In the bolometric mode, noise equivalent power (NEP) down to NEP $\approx 10^{-22} \mathrm{~W} / \sqrt{\mathrm{Hz}}$ at $T_{\text {bath }} \sim 50 \mathrm{mK}$ is predicted and mainly limited by the thermal-fluctuation noise in the detector.

The structure of the manuscript is as follows. In Sec. II, we outline the operation principle and the theoretical background for the temperature-to-phase conversion and its SQUIPT detection. In Sec. III, we discuss the thermal model for the system and the main performance characteristics of the calorimetric operation mode. Section IV discusses the performance characteristics in the bolometric mode, and Sec. V concludes with a discussion.

\section{DETECTOR}

\section{A. Operating principle}

We consider a de SQUID based on two $S-N-S$ Josephson junctions [see Fig. 1(a)]. Each junction consists of two superconducting leads coupled to an $N$ wire of length $L$ through highly transmissive interfaces. The contact with $S$ induces superconducting correlations in the $N$ regions through the proximity effect, which is responsible for the supercurrent flow through the junctions as well as for the modification of the wires' DOS. The transverse dimensions of the wires are assumed to be much smaller than $L$ so that they can be considered as quasi-onedimensional.

Both $S-N-S$ junctions are assumed to be short; i.e., they satisfy the condition $\Delta_{0} \lesssim \hbar D / L^{2}$, where $\Delta_{0}$ is the $S$ energy gap, and $D$ is the diffusion constant of $N$. The above choice is dictated by three main reasons: (i) Drastic reduction of the detector-sensing element volume in order to achieve a substantial improvement of the sensor performance. (ii) The analytical solutions for both the supercurrent and the quasiparticle DOS in the weak links are well known in the short-junction limit, which somewhat simplifies the whole analysis of the detector. (iii) For short junctions, the phase-dependent response of the DOS in the readout junction is maximized leading to enhanced readout transduction sensitivity.

The variables $\varphi_{d}$ and $\varphi_{r}$ denote the macroscopic quantum phase differences across the detector and readout junction, respectively. By neglecting the ring kinetic inductance, it follows from fluxoid quantization that

$$
\varphi_{d}+\varphi_{r}=2 \pi \boldsymbol{\Phi}_{\mathrm{ext}} / \boldsymbol{\Phi}_{0}+2 k \pi,
$$

where $\boldsymbol{\Phi}_{\text {ext }}$ is the applied magnetic flux, $k$ is an integer, and $\boldsymbol{\Phi}_{0} \simeq 2.067 \times 10^{-15} \mathrm{~Wb}$ is the flux quantum.

We suppose the $S$ loop to be backed at a distance $L_{S}$ from the $N-S$ interfaces of the detector junction by Andreev mirrors [20], and superconductors $S_{1}$ with a large energy gap $\Delta_{1} \gg \Delta_{0}$ in good electric contact, so that $S_{1}$ blocks 
out-diffusion of the heat absorbed by the $N$ wire and the $S$ electrodes [see Fig. 1(b)], as quasiparticle energy cannot escape through the $S / S_{1}$ interfaces due to Andreev reflections. The above design for the detector region allows thermal relaxation to occur predominantly via the slower electron-phonon mechanism in the $N$ and $S$ regions. In order to retain the larger kinetic inductance of the shortjunction link determined by $\Delta_{0}$ instead of $\Delta_{1}$, we choose below the length $L_{S} \gg \xi_{0}$ where $\xi_{0}=\sqrt{\hbar D / \Delta_{0}}$ is the coherence length of the weaker superconductor.

The external incident radiation is coupled to the $N$ region via a suitable antenna. If it is coupled as in Fig. 1, the absorbed power is distributed both to the detector and the readout junctions and divided evenly if the junctions are identical. Different designs may also be able to concentrate the power dissipation mainly in the detector junction between the Andreev mirrors. Here, we neglect the impedance of the ring itself, as it is small in the gigahertz to terahertz range compared to the junctions. In the discussion that follows, we ignore heat losses due to part of the power being dissipated in the readout junction.

The antenna impedance matching is influenced by the impedance $Z_{d}$ of the short detector junction, which is qualitatively similar to that of superconductors $[21,22]$. At low temperatures and in the short-junction limit, the dissipative component has a frequency threshold determined by the DOS gap $\varepsilon_{g}=\Delta_{0}\left|\cos \left(\varphi_{d} / 2\right)\right|$ in the junction: $\operatorname{Re} Z_{d}^{-1}(\omega) \sim\left(R_{N}^{d}\right)^{-1} \theta\left(\hbar \omega-2 \varepsilon_{g}\right)+\left(R_{N}^{d}\right)^{-1} e^{-\varepsilon_{g} / k_{B} T}$. At frequencies above the gap, the inductive component is of order $\operatorname{Im} Z_{d}^{-1}(\omega) \sim\left(R_{N}^{d}\right)^{-1} \pi \Delta_{0} /(\hbar \omega)$. As a consequence, we expect that good radiation coupling can be achieved at frequencies $\nu>2 \varepsilon_{g} / h$ by matching the normal-state resistance $R_{N}^{d}$ of the junction to the antenna. At frequencies below the DOS gap, the ability of the proximized normal wire to absorb energy in linear response becomes suppressed at low $T$, which is a generic limitation of superconducting absorber elements. For large enough excitation amplitude, i.e., phase oscillation induced by incoming radiation pulse being $\delta \phi_{d}(t)=e V /(\hbar \omega) \gtrsim 1$, where $V$ is the voltage amplitude across the junction, multiphoton events can contribute to the absorption also at lower frequencies.

It is beneficial for the performance of the device if the readout junction remains at a low temperature compared to the detector junction, even if part of the input power heats it. This condition is enforced by the presence of the superconducting tunnel probe and the lack of Andreev mirrors in the readout junction resulting in electronic heat out-diffusion that is larger by a factor of approximately $e^{\left(\Delta_{1}-\Delta_{0}\right) / k_{B} T} \gg 1$. Depending on the parameters, it is possible to supplement the cooling by an additional tunnel-coupled $N$ cooling fin [23] in the readout junction, but as we discuss in Sec. IVA, this is likely not necessary for the parameters we consider.

\section{B. Model}

In the short-junction limit, the Josephson current $\left(I_{c}^{d, r}\right)$ flowing through the detector and readout weak links at temperature $T$ can be written as $[24,25]$

$$
\begin{aligned}
& I_{c}^{d, r}\left(T, \varphi_{d, r}\right)=\frac{\pi \Delta(T)}{e R_{N}^{d, r}} \cos \left(\frac{\varphi_{d, r}}{2}\right) \int_{\Delta(T) \cos \left(\varphi_{d, r} / 2\right)}^{\Delta(T)} d \varepsilon \\
& \times \frac{1}{\sqrt{\varepsilon^{2}-\Delta^{2}(T) \cos ^{2}\left(\frac{\varphi_{d, r}}{2}\right)}} \tanh \left(\frac{\varepsilon}{2 k_{B} T}\right),
\end{aligned}
$$

where $\Delta(T)$ is the BCS temperature-dependent pairing potential in $S, R_{N}^{d, r}$ is the normal-state resistance of the detector (readout) junction, $k_{B}$ is the Boltzmann constant, and $e$ is the electron charge. In the limit of zero temperature $(T=0)$, Eq. (2) reduces to [24]

$I_{c}^{d, r}\left(0, \varphi_{d, r}\right)=\frac{\pi \Delta(0)}{e R_{N}^{d, r}} \cos \left(\frac{\varphi_{d, r}}{2}\right) \operatorname{artanh}\left[\sin \left(\frac{\varphi_{d, r}}{2}\right)\right]$.

In Eq. (2), $R_{N}^{d, r}=\rho L / A^{d, r}, \rho=\left(\nu_{F} e^{2} D\right)^{-1}$ is the wire resistivity, $\nu_{F}$ the density of states at the Fermi level in $N$, and $A^{d, r}$ is the wire cross section of the detector (readout) Josephson junction. Moreover, in the following, the electron temperature in the detector junction is denoted by $T_{e}$ $\left(T \equiv T_{e}\right)$, whereas that in the readout weak link is supposed to coincide with the lattice temperature $T \equiv T_{\text {bath }}$ (see discussion in Sec. IVA).

The electromagnetic energy $h \nu$ absorbed in the detector junction elevates the temperature $T_{e}$ in $N$ and in the lateral portion of $S$, thereby leading to a decrease of the dissipationless supercurrent $I_{c}^{d, r}$ circulating (for $\boldsymbol{\Phi}_{\text {ext }} \neq 0$ ) in the superconducting loop. The temperature dependence of the junction's critical current is shown in Fig. 2(a) being evaluated from Eq. (2) for $\varphi_{d}=\varphi_{r}=\pi / 2$ by setting $T \equiv T_{e}=T_{\text {bath }}$. Note that the critical supercurrent is almost saturated for $T_{e} \lesssim 0.25 T_{c}$ ( $T_{c}$ is the critical temperature of S) and decreases linearly with the temperature around $T_{e} \approx T_{c}$.

Such a temperature-induced suppression of $I_{c}^{d, r}$ yields a finite variation of the phase drop across both $S-N-S$ junctions owing to the following: (i) conservation of the supercurrent circulating along the $S$ loop and (ii) fluxoid quantization in the interferometer [Eq. (1)]. As a consequence, for a given $\boldsymbol{\Phi}_{\text {ext }}$, the phases $\varphi_{d}$ and $\varphi_{r}$ can be determined for any $T_{e}$ and $T_{\text {bath }}$ from condition (i), i.e., by solving the equation

$$
I_{c}^{d}\left(T_{e}, \varphi_{d}\right)=I_{c}^{r}\left(T_{\mathrm{bath}}, \varphi_{r}\right) .
$$

This equation is at the origin of the TPC process. By defining the parameter $\alpha=R_{N}^{d} / R_{N}^{r}=A^{r} / A^{d}$ as the degree 


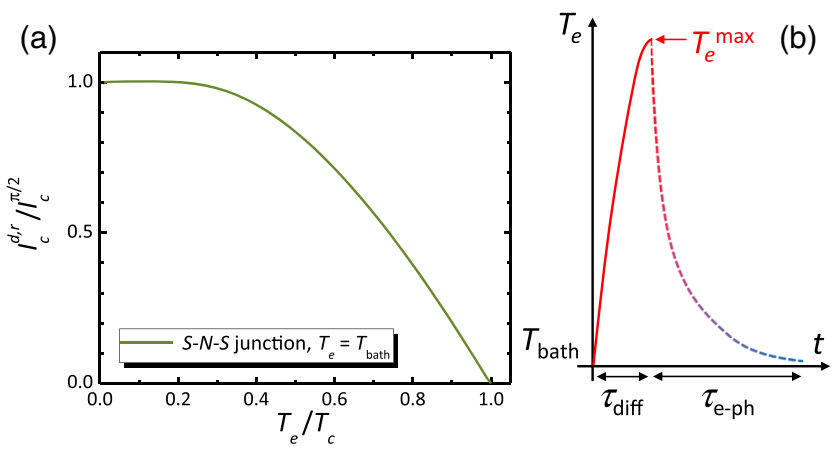

FIG. 2. (a) Josephson current $I^{d, r}$ vs $T_{e}=T_{\text {bath }}$ calculated at $\varphi_{d, r}=\pi / 2 . T_{\text {bath }}$ and $T_{c}$ are the bath and critical temperature of the $S$ loop, respectively, whereas $I_{c}^{\pi / 2}$ is the zero-temperature Josephson current at $\varphi_{d, r}=\pi / 2$. (b) Scheme of time ( $t$ ) evolution of the electronic temperature $T_{e}$ in the detector junction after absorption of a photon at energy $h \nu . T_{e}^{\max }$ is the temperature reached in the weak link after the arrival of a single photonic event. $\tau_{\text {diff }}$ represents the diffusion time across the $N$ wire, whereas $\tau_{e \text {-ph }}$ is the electron-phonon relaxation time. In the present setup, $\tau_{\text {diff }} \ll \tau_{e \text {-ph }}$ due to the short length of the $N$ wire and the low temperature at which the detector is conceived to operate.

of asymmetry of the SQUID junctions, we can solve Eq. (4) for the phase $\varphi_{r}$ existing across the readout junction in order to investigate in detail the full TPC process. We note that the asymmetry parameter can also be written as $\alpha=$ $L_{J}^{d} / L_{J}^{r}$ when both junctions are at the same temperature, where $L_{J}^{d, r}$ is the kinetic inductance of the detector (readout) junction, so that it immediately expresses which junction of the interferometer will mainly determine the phase biasing of the superconducting loop for a given external flux $\boldsymbol{\Phi}_{\text {ext }}$. In particular, for $\alpha \gg 1$ the phase drop along the ring predominantly occurs across the detector junction, whereas in the opposite situation $(\alpha \ll 1)$, the phase drop occurs mainly across the readout weak link.

Figures 3(a) and 3(b) show the phase $\varphi_{r}$ calculated from Eq. (4) as a function of $T_{e}$ and $\alpha$ for $\boldsymbol{\Phi}_{\text {ext }}=0.4 \boldsymbol{\Phi}_{0}$ [Fig. 3(a)] and $\boldsymbol{\Phi}_{\text {ext }}=0.499 \Phi_{0}\left[\right.$ Fig. 3(b)] at $T_{\text {bath }}=0.01 T_{c}$. It turns out that the largest TPC effect occurs by setting $\boldsymbol{\Phi}_{\text {ext }}$ at approximately $0.5 \boldsymbol{\Phi}_{0}$ where the phase response can be very sharp [see Fig. 3(b)]. In particular, by choosing values for $\alpha$ around or slightly less than 1 yields sizable phase modulation amplitudes in the readout junction at higher temperatures $T_{e}$. By contrast, for $\alpha>1$, the modulation amplitude occurs for lower temperatures, although slightly reduced.

The parameter $\alpha$ also controls the phase difference over the detector junction $\varphi_{d}$ and, therefore, the energy gap $\varepsilon_{g}^{d}=\Delta_{0} \cos \left(\varphi_{d} / 2\right)$. As we discuss above, this gap provides a frequency threshold $2 \varepsilon_{g}^{d}$ below which it can be difficult to impedance match the detector junction to an antenna. To reduce $\varepsilon_{g}^{d}$, a larger $\alpha$ should be chosen, which, however, reduces the sensitivity of TPC. Below, we choose this
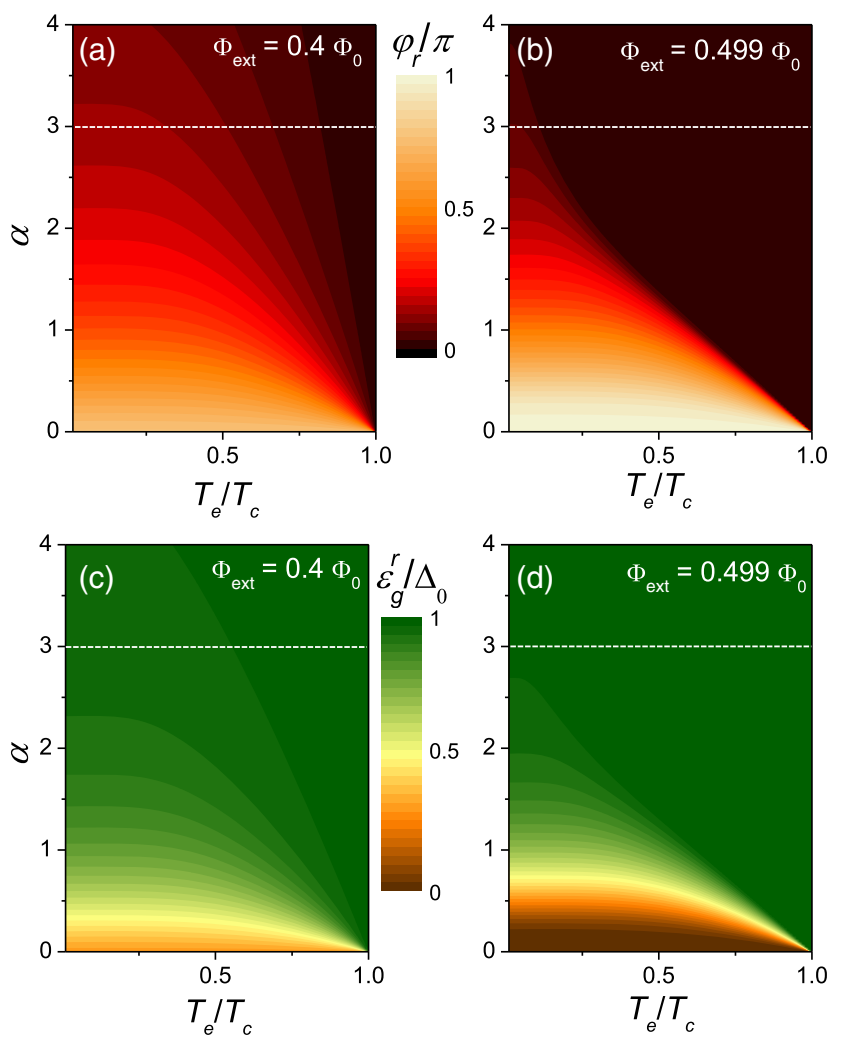

FIG. 3. (a),(b) Contour plot of the phase $\varphi_{r}$ across the readout junction vs $\alpha$ and $T_{e}$ calculated for $\boldsymbol{\Phi}_{\mathrm{ext}}=0.4 \boldsymbol{\Phi}_{0}$ (a) and $\boldsymbol{\Phi}_{\mathrm{ext}}=$ $0.499 \Phi_{0}$ (b) at $T_{\text {bath }}=0.01 T_{c}$. (c),(d) Contour plot of the minigap $\varepsilon_{g}^{r}$ in the $N$ region of the readout junction vs $\alpha$ and $T_{e}$ calculated for $\boldsymbol{\Phi}_{\text {ext }}=0.4 \boldsymbol{\Phi}_{0}$ (c) and $\boldsymbol{\Phi}_{\text {ext }}=0.499 \Phi_{0}(\mathrm{~d})$ at $T_{\text {bath }}=0.01 T_{c}$. Dashed lines in all panels positioned at $\alpha=3$ correspond to the value chosen for the interferometer in all forthcoming calculations unless differently stated. $\Delta_{0}$ denotes the zero-temperature energy gap in corresponding to the critical temperature $T_{c} \simeq \Delta_{0} / 1.764 k_{B}$.

trade-off at $\alpha=3$ (dashed lines in Fig. 3) corresponding to $2 \varepsilon_{g} / h=10 \mathrm{GHz}$. If a higher frequency threshold is acceptable, better performance characteristics can be achieved by values closer to $\alpha=1$.

\section{Readout weak-link behavior}

The temperature-induced suppression of the critical current may thereby yield a variation of phase drop $\left(\delta \varphi_{r}\right)$ across the readout junction. Measuring $\delta \varphi_{r}$ with a suitable setup enables us to assess with accuracy the electronic temperature $T_{e}$ and, hence, the radiation absorbed by the $S-N-S$ weak link of the detector. Yet, owing to the proximity effect, $\varphi_{r}$ affects the spectral characteristics of the corresponding $N$ region as well, for instance, by determining the exact shape of the local quasiparticle DOS in the $N$ wire. In the following, we show that probing the phase-induced variations of the DOS with a SQUIPT implemented in the readout junction is a 
simple and effective way to get direct and detailed information about $T_{e}$-driven phase changes.

The SQUIPT [shown on the upper part of the scheme of Fig. 1(a)] consists of a superconducting tunnel junction with normal-state resistance $R_{p}$ coupled to the middle of the $N$ wire of the readout junction [17]. So far, SQUIPTs have been implemented in a few geometrical configurations $[17,26,27]$ and with different material combinations $[17,28,29]$. For the sake of clarity, we assume here the probing electrode (of width $w$ ) to be made of the same superconducting material $S$ as the SQUID ring. $\varphi_{r}$ changes induced by temperature $T_{e}$ variations in the detector junction affect the readout $N$ wire DOS and thereby the current $I_{p}$ vs voltage characteristic of the superconducting tunnel junction biased at voltage $V$ [see Fig. 1(a)].

Let us now analyze the $N$ wires DOS $\left(\mathcal{N}_{N}^{d, r}\right)$ in the $S-N-S$ junctions. In the short-junction limit, which is the relevant one for the present case, we have the well-known result (cf. e.g., Refs. [25,30]):

$$
\begin{aligned}
\mathcal{N}_{N}^{d, r}\left(x, \varphi_{d, r}, \varepsilon, T\right) & \\
= & \operatorname{Re} \sqrt{\frac{(\varepsilon+i \Gamma)^{2}}{(\varepsilon+i \Gamma)^{2}-\Delta^{2}(T) \cos ^{2} \frac{\varphi_{d, r}}{2}}} \\
& \times \cosh \left(\frac{2 x-L}{L} \operatorname{arcosh} \sqrt{\frac{(\varepsilon+i \Gamma)^{2}-\Delta^{2}(T) \cos ^{2} \frac{\varphi_{d, r}}{2}}{(\varepsilon+i \Gamma)^{2}-\Delta^{2}(T)}}\right),
\end{aligned}
$$

where $\varepsilon$ is the energy relative to the chemical potential of the superconductors, $x \in[0, L]$ is the spatial coordinate along the $N$ wires, and $\Gamma$ is the Dynes parameter accounting for broadening in $S$.

$\mathcal{N}_{N}^{d, r}$ exhibits a minigap $\varepsilon_{g}^{d, r}\left(\varphi_{d, r,} T\right)=\Delta(T)\left|\cos \left(\varphi_{d, r} / 2\right)\right|$ for $|\varepsilon| \leq \varepsilon_{g}^{d, r}$ whose amplitude depends on $\varphi_{d, r}$, and is spatially constant along the $N$ wires. In particular, $\varepsilon_{g}^{d, r}=\Delta$ for $\varphi_{d, r}=0$ and decreases by increasing the value of the phase, vanishing at $\pi$. Therefore, the quasiparticle spectrum in the $N$ region can vary from that of a gapped superconducting material (for $\varphi_{d, r}=0$ ) to that of a gapless normal conductor (at $\varphi_{d, r},=\pi$ ) just by changing the phase across the weak link.

The impact of the electronic temperature $T_{e}$ of the detector on the DOS in the readout $N$ weak link is displayed in Figs. 3(c) and 3(d) where the minigap amplitude $\varepsilon_{g}^{r}$ is plotted as a function of $T_{e}$ and $\alpha$ for $\boldsymbol{\Phi}_{\text {ext }}=0.4 \boldsymbol{\Phi}_{0}$ [Fig. 3(c)] and $\boldsymbol{\Phi}_{\text {ext }}=0.499 \boldsymbol{\Phi}_{0}$ [Fig. 3(d)], both calculated at $T_{\text {bath }}=0.01 T_{c}$. These results show that the minigap behavior is qualitatively similar to that of $\varphi_{r}$ and confirm that for $\alpha=3$ it is possible to obtain sufficient $T_{e}$-induced modulation of $\varepsilon_{g}^{r}$. The above value for $\alpha$ is set in all forthcoming calculations, unless differently stated, to evaluate the response and performance of the nanodetector.

\section{SQUIPT response}

Let us now turn to discussing the behavior of the SQUIPT current vs voltage characteristics which allow us to understand how to exactly operate the superconducting interferometer for radiation detection in the present setup. The current flowing through the superconducting tunnel probe of the SQUIPT in the readout junction is dominated by quasiparticles and can be written as [31]

$$
\begin{aligned}
I_{p}(V)= & \frac{1}{e w R_{p}} \int_{[(L-w) / 2]}^{[(L+w) / 2]} d x \\
& \times \int_{-\infty}^{\infty} d \varepsilon \mathcal{N}_{N}^{r}\left(x, \varepsilon, \varphi_{r}\right) \mathcal{N}_{S}^{p}(\tilde{\varepsilon}) F(\varepsilon, \tilde{\varepsilon}),
\end{aligned}
$$

where $\mathcal{N}_{S}^{p}\left(\varepsilon, T_{e}\right)=\left|\operatorname{Re}\left[(\varepsilon+i \Gamma) / \sqrt{(\varepsilon+i \Gamma)^{2}-\Delta^{2}\left(T_{e}\right)}\right]\right|$ is the BCS normalized DOS of the $S$ probe electrode at temperature $T_{\text {bath }}, \tilde{\varepsilon}=\varepsilon-e V, F(\varepsilon, \tilde{\varepsilon})=\left[f_{0}(\tilde{\varepsilon})-f_{0}(\varepsilon)\right]$, $f_{0}(\varepsilon)$ is the Fermi-Dirac energy distribution function, and $R_{p}$ is the normal-state tunneling resistance of the probing junction. In the following calculations, we assume for simplicity that the superconductor forming the probing electrode is identical to that realizing the loop so that $\mathcal{N}_{S}^{p}(\varepsilon, T)=\mathcal{N}_{S}^{d}(\varepsilon, T)$. Moreover, we set $w=L / 3$ and $R_{p}=10^{5} \Omega$ as characteristic parameters of the SQUIPT readout. For calorimetric operation, we also assume the measurement of the current is possible on bandwidths comparable to the relevant inverse thermal relaxation time of the detector junction (see below); the possible fast readout schemes are discussed in Refs. $[18,19]$.

Figure 4(a) illustrates the general behavior of the lowtemperature SQUIPT current vs voltage characteristics $I_{p}(V)$ at $\boldsymbol{\Phi}_{\mathrm{ext}}=0.499 \boldsymbol{\Phi}_{0}$ in the absence of radiation (i.e., $h \nu=0, T_{e}=T_{\text {bath }}$ ), and for nonzero radiation ( $\left.h \nu \neq 0, T_{e}>T_{\text {bath }}\right)$ heating the detector junction. In particular, the onset of large quasiparticle tunneling occurs at an energy corresponding to the sum of the gaps in the superconducting probe and the $N$ proximity layer, as expected for a tunneling process through a $S-I-S$ tunnel junction [32]. Therefore, in the absence of radiation, this onset appears at $e V_{0}=\Delta(T)+\varepsilon_{g}^{r}(T, \nu=0)$, whereas under the effect of radiation of energy $h \nu$, the onset occurs at $e V_{\nu}=\Delta(T)+\varepsilon_{g}^{r}(T, \nu)$. Moreover, $2 \Delta(T) / e \geq V_{\nu}>V_{0}$ since $\Delta(T) \geq \varepsilon_{g}^{r}(T, \nu)>\varepsilon_{g}^{r}(T, \nu=0)$ as a consequence of heating in the detector junction originating from absorption of radiation [see Figs. 3(c) and 3(d)]. From these results, it follows that by biasing the SQUIPT at voltage $V^{*}$ (with $V_{0} \lesssim V^{*} \lesssim V_{\nu}$ ), the absorption of a photon will yield a reduction $(\delta I)$ of the current $I_{p}$ flowing through the tunneling probe. As a consequence, a direct radiation readout can be performed with the SQUIPT by a simple measurement of its current at fixed bias voltage.

The impact of asymmetry of the two $S-N-S$ Josephson junctions forming the SQUID on the SQUIPT characteristics is displayed in Fig. 4(b), which shows $I_{p}$ vs $V$ 

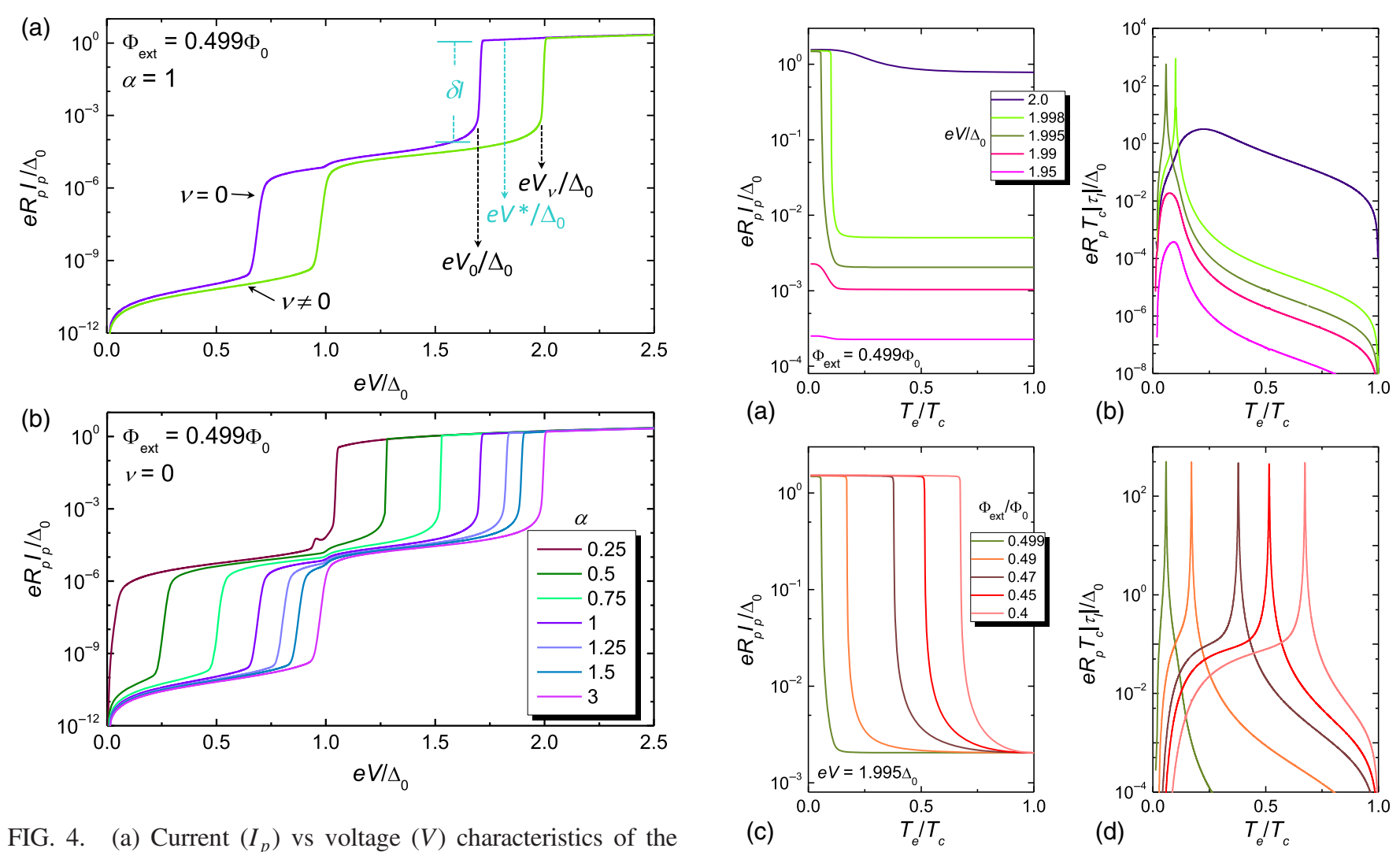

FIG. 4. (a) Current $\left(I_{p}\right)$ vs voltage $(V)$ characteristics of the SQUIPT readout junction in the absence $\left(T_{e}=T_{\text {bath }}, \nu=0\right)$ and for finite incoming radiation $\left(T_{e}>T_{\text {bath }}, \nu \neq 0\right)$ calculated for $\boldsymbol{\Phi}_{\text {ext }}=0.499 \boldsymbol{\Phi}_{0}, \alpha=1$, and $T_{\text {bath }}=0.01 T_{c}$. The voltage onset of large quasiparticle tunneling for zero $(h \nu=0)$ and finite frequency $(h \nu \neq 0)$ are indicated (black dashed arrows). The bias voltage of the SQUIPT readout junction is denoted by $V^{*}$, whereas $\delta I$ indicates the current variation due to the absorption of a photon of energy $h \nu$. (b) $I_{p}$ vs $V$ calculated for $\boldsymbol{\Phi}_{\text {ext }}=0.499 \Phi_{0}, T_{\text {bath }}=0.01 T_{c}, \nu=0$, and for several values of $\alpha$. The degree of asymmetry of the interferometer determines the onset of large quasiparticle tunneling in the absence of radiation.

calculated for selected values of $\alpha$ at $\boldsymbol{\Phi}_{\text {ext }}=0.499 \boldsymbol{\Phi}_{0}$ and $\nu=0$. The figure shows that increasing $\alpha$ leads to $V_{0}$ approaching $2 \Delta_{0}$, as expected from the increase of $\varepsilon_{g}^{r}$ in the readout junction [see Figs. 3(c) and 3(d)]. Therefore, a precise tuning of the SQUIPT working voltage $V^{*}$ can, in principle, be achieved by setting the asymmetry of the junctions forming the SQUID.

For the value $\alpha=3$ used in the remainder of the text, at $T_{e}=T_{\text {bath }}=0.01 \Delta_{0}$ and $\boldsymbol{\Phi}_{\text {ext }}=0.499 \boldsymbol{\Phi}_{0}, \quad \varphi_{r} \approx 0.07 \pi$, and the voltage threshold for $\nu=0$ [see Fig. 4(a)] resides at $e V_{0} \approx 1.994 \Delta_{0}$.

\section{E. Temperature-to-current conversion and noise analysis}

Hereafter, we describe the behavior of the SQUIPT corresponding to a temperature-to-current transducer, i.e., the ability of the system to convert a temperature variation

FIG. 5. (a) Current $I_{p}$ vs temperature $T_{e}$ characteristics of the SQUIPT readout junction calculated for a few values of $V$ at $\boldsymbol{\Phi}_{\text {ext }}=0.499 \Phi_{0}$. (b) Absolute value of the temperature-tocurrent transfer function $\left|\tau_{I}\right|$ vs $T_{e}$ calculated for the same parameters as in panel (a). (c) $I_{p}$ vs $T_{e}$ characteristics calculated for a few values of $\boldsymbol{\Phi}_{\text {ext }}$ at $V=1.995 \Delta_{0} / e$. (d) Absolute value of the temperature-to-current transfer function $\left|\tau_{I}\right|$ vs $T_{e}$ calculated for the same parameters as in panel (c). In all these calculations, we set $\alpha=3$ and $T_{\text {bath }}=0.01 T_{c}$.

$\delta T_{e}$ in the detector weak link into a current change $\delta I$ in the readout tunnel junction.

Figure 5(a) shows the dependence of the current $I_{p}$ through the probing junction on the temperature $T_{e}$ for different values of $V$ by keeping fixed the external flux $\boldsymbol{\Phi}_{\text {ext }}=0.499 \boldsymbol{\Phi}_{0}$ for $\alpha=3$ and $T_{\text {bath }}=0.01 T_{c}$. In particular, the current turns out to be strongly dependent on $T_{e}$ for specific values of the biasing voltage; in particular, we note that for $V=V^{*}=1.995 \Delta_{0} / e$ and $V=V^{*}=1.998 \Delta_{0} / e$, $I_{p}$ can vary significantly for temperatures $T_{e}$ in the range of approximately $0.05 T_{c}$ to $0.2 T_{c}$. The above $V^{*}$ values stem from the chosen parameters of the structure, and will lead to a high sensitivity for radiation detection. By contrast, for other bias-voltage values, the current response is somewhat moderate in the whole range of temperatures.

A figure of merit which is useful to characterize the readout weak-link performance is represented by the temperature-to-current transfer function $\tau_{I}=\partial I_{p} / \partial T_{e}$, which is shown in Fig. 5(b) for the same parameters as in Fig. 5(a). For $V=V^{*}=1.995 \Delta_{0} / e$ and $V=V^{*}=1.998 \Delta_{0} / e, \tau_{I}$ 
obtains the largest values in the range of approximately $0.05 T_{c}$ to $0.2 T_{c}$, which is expected from the corresponding behavior of the current in the same temperature window.

Figure 5(c) displays the dependence of $I_{p}$ on $T_{e}$ for different values of the applied magnetic flux $\boldsymbol{\Phi}_{\text {ext }}$ by keeping fixed the bias voltage $V=1.995 \Delta_{0} / e$ for $\alpha=3$ and $T_{\text {bath }}=0.01 T_{c}$. Approaching the $0.5 \boldsymbol{\Phi}_{0}$ flux leads to a stronger response of tunneling current through the readout junction. On the other side, values of $\boldsymbol{\Phi}_{\text {ext }}$ far away from $0.5 \boldsymbol{\Phi}_{0}$ correspond in general to a weaker dependence of $I_{p}$ on the temperature. The corresponding transfer functions are shown in Fig. 5(d) and are calculated for the same parameters as in Fig. 5(c).

The intrinsic temperature sensitivity (temperature noise) per unit bandwidth of the probe junction $\left(s_{T}\right)$ is related to its current-noise spectral density $\left(\mathcal{S}_{I}\right)$ as

$$
s_{T}=\frac{\sqrt{\mathcal{S}_{I}}}{\left|\tau_{I}\right|}
$$

where $\tau_{I}$ is the temperature-to-current transfer function discussed before. The low-frequency current-noise spectral density of the tunnel probe is [33]

$$
\mathcal{S}_{I, \text { tun }}(V)=2 e I_{p}(V) \operatorname{coth}\left(\frac{e V}{2 k_{B} T_{\text {bath }}}\right) \text {. }
$$

We note that Eq. (8) describes both regimes of shot noise, i.e., $k_{B} T_{\text {bath }} \ll e V$, and thermal noise, i.e., $e V \ll k_{B} T_{\text {bath }}$, and holds in the tunneling limit.

In Fig. 6(a), we show the temperature noise $s_{T}$ vs $T_{e}$ calculated for selected values of $V$ at $\boldsymbol{\Phi}=0.499 \boldsymbol{\Phi}_{0}$. The maximum temperature sensitivity is obtained for $V \sim 1.95-2.0 \Delta_{0} / e$, where the temperature noise can be of the order of $10^{-8} \mathrm{~K} / \sqrt{\mathrm{Hz}}$. Figure 6(b) displays the temperature noise vs $T_{e}$ calculated for a few values of the
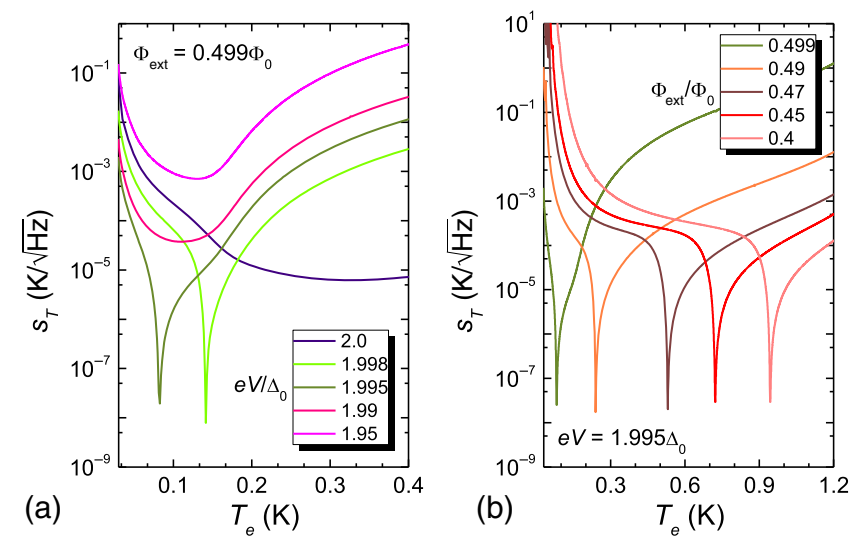

FIG. 6. (a) Temperature sensitivity $s_{T}$ vs temperature $T_{e}$ calculated for a few values of $V$ at $\boldsymbol{\Phi}_{\text {ext }}=0.499 \Phi_{0}$. (b) $s_{T}$ vs $T_{e}$ calculated for a few values of $\boldsymbol{\Phi}_{\mathrm{ext}}$ at $V=1.995 \Delta_{0} / e$. In all calculations, we set $\alpha=3$ and $T_{\text {bath }}=0.01 T_{c}$. external flux at $V=1.995 \Delta_{0} / e$. In particular, $s_{T}$ is minimized at different temperatures depending on the specific value of $\boldsymbol{\Phi}_{\text {ext }}$ and obtains values as low as a few tens of $\mathrm{nK} / \sqrt{\mathrm{Hz}}$ at a temperature range tunable over $T=10 \mathrm{mK}$ to $1 \mathrm{~K}$ with the choice of $\boldsymbol{\Phi}_{\text {ext }}$.

\section{NANOCALORIMETER OPERATION}

\section{A. Thermal model}

The operation principle of single-photon detection (i.e., operation as a calorimeter) based on the TPC effect can be understood by inspecting the scheme displayed in Fig. 2(b) which shows a sketch of time evolution of the electronic temperature $T_{e}$ in the detector weak link after the arrival of a photonic event. In particular, we assume that depending on the photon energy $h \nu, T_{e}$ is increased with respect to $T_{\text {bath }}$ up to $T_{e}^{\max }$ uniformly along the wire over a timescale set by fast timescales, e.g., the diffusion time $\tau_{\text {diff }}=L^{2} / D$ (see red full line) and $L C$ time of the superconducting loop. The diffusion time with the typical parameters chosen for our $S-N-S$ junctions is of the order of approximately $10^{-12} \mathrm{~s}$. After the initial absorption, the electronic temperature $T_{e}$ relaxes toward the bath temperature over a timescale set by the electron-phonon relaxation time $\tau_{e-\mathrm{ph}}$. For instance, in the normal state, this time is given by $\tau_{e \text {-ph }} \approx\left[\left(k_{B}^{2} \nu_{F}\right) /(0.34 \Sigma)\right] T_{\text {bath }}^{-3}$, where $\Sigma$ is the electronphonon coupling constant. By setting, for instance, $\Sigma=5 \times 10^{8} \mathrm{Wm}^{-3} \mathrm{~K}^{-5}$ typical of silver (Ag), we obtain $\tau_{e-\mathrm{ph}} \in\left[10^{-4}-10^{-7}\right] \mathrm{s}$ for $T_{e} \in\left[10^{-1}-1\right] \mathrm{K}$ so that $\tau_{\text {diff }} \ll$ $\tau_{e \text {-ph }}$ [see dashed line in Fig. 1(c)]. As we shall argue, in our system, thanks to superconducting correlations induced in the $N$ regions, $\tau_{e \text {-ph }}$ can be longer than that in the normal state.

Consider now an event where the detector junction absorbs energy $\delta U=h \nu$. The energy $\delta U\left(T_{e}, T_{\text {bath }}, \boldsymbol{\Phi}_{\text {ext }}\right)$ required for increasing the temperature of the detector junction from $T_{\text {bath }}$ to $T_{e}$ consists of heat $\delta Q^{d}$ of the quasiparticles in the detector junction and work $\delta W=$ $\delta W^{d}+\delta W^{r}$ required for changing the phase differences over the junctions $\varphi_{d, r}^{(0)} \mapsto \varphi_{d, r}^{(1)}$. The energy required is bounded by free-energy differences $\delta W^{r, d} \geq \delta F^{r, d}$. Below, we assume that $\delta W^{r}=\delta F^{r}$ and that the detector does not exchange heat with other systems (on the short timescales): in the real device, the energy required can be larger due to some heat $\delta Q^{r} \geq 0$ entering the readout junction and exiting the heat sinks. For a device where the detector junction is impedance matched to the antenna better than the readout, we expect $\delta Q^{r}<\delta Q^{d}$ so that most of the absorbed heat is useful for detection.

In the above model, we have

$$
\begin{aligned}
\delta U\left(T_{e}, T_{\text {bath }}, \boldsymbol{\Phi}_{\mathrm{ext}}\right)= & U^{d}\left(\varphi_{d}^{(1)}, T_{e}\right)-U^{d}\left(\varphi_{d}^{(0)}, T_{\mathrm{bath}}\right) \\
& +F^{r}\left(\varphi_{r}^{(1)}, T_{\mathrm{bath}}\right)-F^{r}\left(\varphi_{r}^{(0)}, T_{\mathrm{bath}}\right) .
\end{aligned}
$$


Above, the phases $\varphi_{r, d}^{(1)}=\varphi_{r, d}\left(T_{e}, T_{\mathrm{bath}}, \boldsymbol{\Phi}_{\mathrm{ext}}\right)$ and $\varphi_{r, d}^{(0)}=$ $\varphi_{r, d}\left(T_{\text {bath }}, T_{\text {bath }}, \boldsymbol{\Phi}_{\text {ext }}\right)$ are the functions of the temperature and flux that we discuss in Sec. II B. The effective total electronic heat capacity for constant $\boldsymbol{\Phi}_{\text {ext }}$ is then defined as

$$
\begin{array}{r}
C_{\mathrm{tot}}^{e}\left(T_{e}, T_{\mathrm{bath}}\right)=\frac{d}{d T_{e}} \delta U\left(T_{e}, T_{\mathrm{bath}}, \boldsymbol{\Phi}_{\mathrm{ext}}\right) \\
=T_{e} \frac{d}{d T_{e}} S^{d}\left[T_{e}, \varphi_{d}^{(1)}\left(T_{e}, T_{\mathrm{bath}}, \boldsymbol{\Phi}_{\mathrm{ext}}\right)\right],
\end{array}
$$

and it accounts for the readjustment of the phases. The relation to the quasiparticle entropy $S^{d}=-\partial_{T} F^{d}$ of the detector follows from Eq. (9), $U^{d, r}=F^{d, r}+T S^{d, r}$ and the current balance $I^{d}-I^{r}=\partial_{\varphi_{d}} F^{d}\left(\varphi_{d}^{(1)}, T_{e}\right)-\partial_{\varphi_{r}} F^{r}\left(\varphi_{r}^{(1)}, T_{\text {bath }}\right)=0$.

The entropy for short $S-N-S$ junctions is to a good approximation [34] given by

$$
\begin{aligned}
S^{d}(\varphi, T) \simeq & \left(\mathcal{V}_{S}^{d}+\mathcal{V}_{N}^{d}\right) \mathcal{S}_{\mathrm{BCS}}(T) \\
& -\frac{\hbar}{2 e} \int_{0}^{\varphi} d \varphi^{\prime} \partial_{T} I_{c}^{d}\left(\varphi^{\prime}, T\right),
\end{aligned}
$$

where the first term also accounts for the phase-independent heat capacity of the superconducting $S$ part of the detector. The second term corresponds to the quasiparticle states bound at the junction; the result automatically satisfies the thermodynamic relation $\partial_{\varphi} S=-[\hbar /(2 e)] \partial_{T} I_{c}$. The bulk superconductor entropy density is

$$
\begin{aligned}
\mathcal{S}_{\mathrm{BCS}}(T)= & -2 \nu_{F}^{S} k_{B} \int_{-\infty}^{\infty} d \varepsilon \mathcal{N}_{\mathrm{BCS}}[T, \Delta(T)] \\
& \times f_{0}(\varepsilon, T) \ln \left[f_{0}(\varepsilon, T)\right] .
\end{aligned}
$$

From the above, we can obtain the relations $\delta U\left(T_{e}, T_{\text {bath }}, \boldsymbol{\Phi}_{\text {ext }}\right)$ and $T_{e}\left(\delta U, T_{\text {bath }}, \boldsymbol{\Phi}_{\text {ext }}\right)$ for given system parameters.

We now fix the parameters for the hybrid detector in Fig. 1(b). For the normal part, we choose a 10-nm-thick, 10-nm-wide $\mathrm{Ag}$ wire with $L=200 \mathrm{~nm}$ (volume $\left.\mathcal{V}_{N}=2 \times 10^{-23} \mathrm{~m}^{3}=200 \times 10 \times 10 \mathrm{~nm}^{3}\right)$. For the superconducting part $(S)$, we consider aluminum (Al) with $T_{c}=1.4 \mathrm{~K}, \Delta_{0}=1.764 k_{B} T_{c}$, and select volume $\mathcal{V}_{S}=$ $2 \times 10^{-21} \mathrm{~m}^{3}=2\left(500 \times 20 \times 100 \mathrm{~nm}^{3}\right) \quad$ corresponding to $L_{S}=500 \mathrm{~nm}$. The densities of states are $\nu_{F}=$ $1.0 \times 10^{47} \mathrm{~J}^{-1} \mathrm{~m}^{-3}, \nu_{F}^{S}=2.15 \times 10^{47} \mathrm{~J}^{-1} \mathrm{~m}^{-3}$, and $D=$ $0.01 \mathrm{~m}^{2} \mathrm{~s}^{-1}$. This corresponds to normal-state resistance of $R_{N} \simeq 80 \Omega$, but other material choices providing better impedance matching are also possible.

\section{B. Temperature response of the detector weak link}

It is interesting to show, first of all, the behavior of the total electronic heat capacity $C_{\mathrm{tot}}^{e}$ of the detector junction, as it determines the temperature response of the sensor. The total heat capacity displayed in Fig. 7(a) is calculated vs
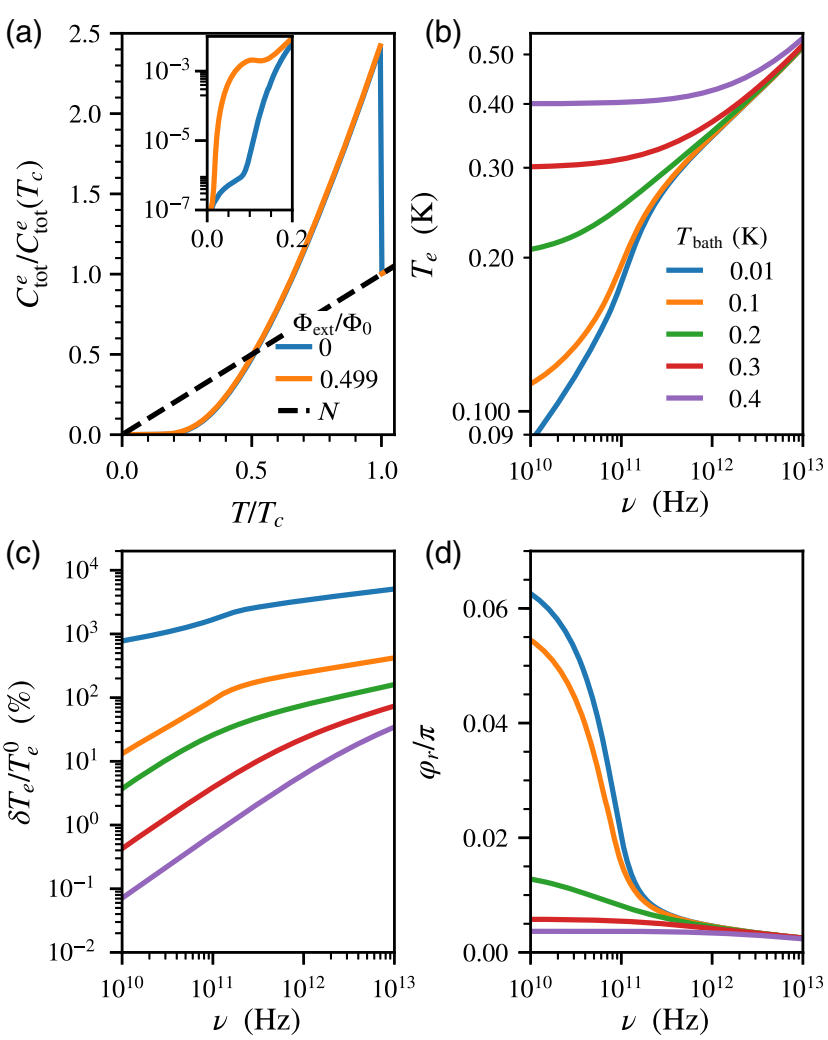

FIG. 7. (a) Behavior of the total electronic heat capacity $C_{\text {tot }}^{e}$ in the detector junction vs temperature $T_{e}$ calculated at $10 \mathrm{mK}$ for two values of the applied magnetic flux. Dashed line represents the total electronic heat capacity when the system is in the normal state. The inset shows an enlargement of $C_{\text {tot }}^{e}$ in the lowtemperature regime. (b) Electronic temperature in the detector junction $T_{e}$ vs $\nu$ calculated for several values of $T_{\text {bath }}$ at $\boldsymbol{\Phi}_{\text {ext }}=0.499 \Phi_{0}$. (c) Relative variation of the electronic temperature $\delta T_{e} / T_{e}^{0}$ vs $\nu$ calculated for the same parameters as in panel (b). (d) Phase $\varphi_{r}$ across the readout weak link vs absorbed energy $h \nu$ calculated for a few $T_{\text {bath }}$ values at $\boldsymbol{\Phi}_{\text {ext }}=0.499 \Phi_{0}$ and $\alpha=3$.

temperature $T_{e}$ for two relevant values of the applied magnetic flux at $T_{\text {bath }}=10 \mathrm{mK}$. The general behavior of $C_{\text {tot }}^{e}$ is the one typical of a BCS superconductor; i.e., it is characterized by an amplitude exponentially suppressed with respect to that in the normal state at low $T_{e}$ (i.e., for $T_{e} \lesssim 0.25 T_{c}$ ) and a sizable discontinuity at the critical temperature $T_{c}$. The exponential suppression of $C_{\mathrm{tot}}^{e}$ at low $T_{e}$ is at the origin of the high sensitivity of detection for microwave photons typical of our setup.

Figure 7(b) shows the final electronic temperature $T_{e}$ in the detector junction as a function of energy absorbed $(h \nu)$ from the incoming photon calculated from Eq. (9) for several values of $T_{\text {bath }}$ at $\boldsymbol{\Phi}=0.499 \boldsymbol{\Phi}_{0}$. We notice, first of all, the sizable enhancement of the temperature, typically occurring below approximately $100 \mathrm{GHz}$, which can be achieved in the junction at low bath temperature, in particular, for $T_{\text {bath }} \lesssim 100 \mathrm{mK}$. This enhancement stems predominantly from both the exponentially suppressed amplitude of the junction electronic heat capacity $C_{\mathrm{tot}}^{e}$ 
and from the reduced volume of the $N$ wire, which is peculiar for the present setup. We emphasize that for a bath temperature of $10 \mathrm{mK}, T_{e}$ obtains values as high as approximately $90 \mathrm{mK}$ for $10-\mathrm{GHz}$ photons and up to approximately $150 \mathrm{mK}$ at $100 \mathrm{GHz}$. For larger $T_{\text {bath }}$, the increase of $T_{e}$ is less pronounced below $100 \mathrm{GHz}$ owing to the temperature-driven enhancement of the electronic heat capacity and becomes sizable only at frequencies exceeding $1 \mathrm{THz}$.

The relative variation of the temperature $\delta T_{e} / T_{e}^{0}=$ $\left[T_{e}(h \nu)-T_{e}(0)\right] / T_{e}(0)$ versus $h \nu$ for the same values of $T_{\text {bath }}$ as in Fig. 7(b) and $\boldsymbol{\Phi}=0.499 \boldsymbol{\Phi}_{0}$ is displayed in Fig. 3(c). In the present setup, $\delta T_{e} / T_{e}^{0}$ at approximately $800 \%$ for $10-\mathrm{GHz}$ photons and approximately $4000 \%$ for $10-\mathrm{THz}$ photons can be obtained at $10-\mathrm{mK}$ bath temperature. By increasing $T_{\text {bath }}, \delta T_{e} / T_{e}^{0}$ gets reduced reaching approximately $10 \%$ for $10-\mathrm{GHz}$ photons and approximately $300 \%$ for $10-\mathrm{THz}$ radiation at $T_{\text {bath }}=100 \mathrm{mK}$. These results for substantial temperature variations in the weak link suggest that a large signal-to-noise ratio can be achieved with a TPC-based single-photon detector in the microwave frequency range.

The evolution of the phase $\varphi_{r}$ across the readout junction as a function of the photon energy $h \nu$ is shown in Fig. 7(d) for several bath temperatures at $\boldsymbol{\Phi}=0.499 \boldsymbol{\Phi}_{0}$. Note that at low $T_{\text {bath }}$, the phase across the weak link starts to be reduced already for a few tens of gigahertz, whereas at a higher bath temperature, a reduction of $\varphi_{r}$ occurs only for larger frequencies. This behavior stems from the fact that at low bath temperature, it is easy to enhance the electron temperature also at low photon energy due to suppressed electronic heat capacity, with the following reduction of circulating supercurrent and thereby of phase drop across the readout junction [see Figs. 3(a) and 3(b)]. By contrast, at higher $T_{\text {bath }}$, the total heat capacity is larger so that higher photon energies are required to change the junction temperature appreciably. As a consequence, the reduction of $\varphi_{r}$ is less pronounced.

\section{Performance: Signal-to-noise ratio and resolving power}

In the operation as a calorimeter, i.e., in the pulsed detection mode, we define the SNR of the detector as

$$
\frac{S}{N}\left[T_{e}(h \nu)\right]=\frac{\left|I_{p}\left(V, T_{\text {bath }}\right)-I_{p}\left[V, T_{e}(h \nu)\right]\right|}{\sqrt{\mathcal{S}_{I, \text { tot }}} \sqrt{\omega}},
$$

where $I_{p}\left(V, T_{\text {bath }}\right)$ is current flowing through the SQUIPT biased at voltage $V$ in the idle state, $I_{p}\left[V, T_{e}(h \nu)\right]$ is the current through the SQUIPT after the absorption of energy $h \nu, \mathcal{S}_{I, \text { tot }}$ is the current-noise spectral density in the absence of radiation, and $\omega$ is the SQUIPT measurement bandwidth. Note that in the denominator of Eq. (13), the current-noise spectral density of the tunnel junction is evaluated in the idle state $(h \nu=0)$, where the tunneling noise contribution to $\mathcal{S}_{I, \text { tun }}$ is larger than for $h \nu \neq 0$ [see Fig. 4(a) and Eq. (8)]. In the calorimeter operation mode, the expression is appropriate for measurement bandwidths $\omega \gtrsim 2 \pi / \tau$, where $\tau$ is the characteristic timescale for the relaxation of the system back to equilibrium after absorption of a photon. In general, $\tau$ is determined by the relaxation processes occurring in the weak link, and, in the present setup, the predominant energy relaxation mechanism stems from electron-phonon interaction (see below). Moreover, we also note that for the tunneling noise contribution $S / N \propto 1 / \sqrt{R_{p}}$ so that a SQUIPT tunnel probe with lower resistance is, in general, beneficial in order to maximize the SNR.

After energy absorption, the equation governing time evolution of the temperature $T_{e}$ in the sensor weak link can be written as

$$
C_{\mathrm{tot}}^{e}\left(T_{e}, T_{\mathrm{bath}}, \boldsymbol{\Phi}_{\mathrm{ext}}\right) \frac{d T_{e}}{d t}=-\dot{Q}_{e-\mathrm{ph}}^{\mathrm{tot}}\left(T_{e}, T_{\mathrm{bath}}\right),
$$

where $\dot{Q}_{e \text {-ph }}^{\text {tot }}\left(T_{e}, T_{\text {bath }}\right)=\dot{Q}_{e \text {-ph }}^{N}\left(T_{e}, T_{\text {bath }}\right)+\dot{Q}_{e \text {-ph }}^{S}\left(T_{e}, T_{\text {bath }}\right)$ is the total heat flow between electrons and lattice phonons in the detector region. Here, $\dot{Q}_{e \text {-ph }}^{N}$ is heat exchanged in the $N$ region, whereas $\dot{Q}_{e \text {-ph }}^{S}$ is the one exchanged in the lateral $S$ portions of the detector. $\dot{Q}_{e \text {-ph }}^{i}\left(T_{e}, T_{\text {bath }}\right.$ ) (with $i=N, S$ ) is given by $[35,36]$

$$
\begin{aligned}
\dot{Q}_{e-\mathrm{ph}}^{i}= & -\frac{\Sigma_{i} \mathcal{V}_{i}}{96 \zeta(5) k_{B}^{5}} \int_{-\infty}^{\infty} d E E \int_{-\infty}^{\infty} d \varepsilon M_{E, E+\varepsilon}\left[\Delta_{i}\left(T_{e}\right), \Gamma_{i}\right] \\
& \times \varepsilon|\varepsilon|\left[\operatorname{coth}\left(\frac{\varepsilon}{2 k_{B} T_{\text {bath }}}\right)\left(f_{E}-f_{E+\varepsilon}\right)-f_{E} f_{E+\varepsilon}+1\right],
\end{aligned}
$$

where $f_{E}\left(E, T_{e}\right)=\tanh \left[E /\left(2 k_{B} T_{e}\right)\right], M_{E, E^{\prime}}^{i}=\mathcal{N}_{i}^{d}(E) \mathcal{N}_{i}^{d}\left(E^{\prime}\right)-$ $\operatorname{Re}\left[\mathcal{F}_{i}^{d}(E) \mathcal{F}_{i}^{d}\left(E^{\prime}\right)^{*}\right]$, and $\Sigma_{i}$ is the electron-phonon coupling constant in the $N(S)$ region. Here, $\mathcal{F}$ are anomalous spectral densities, which for BCS superconductors, have the form

$$
\mathcal{F}_{S}(E)=\Delta \operatorname{Re} \frac{\operatorname{sgn}(E)}{\sqrt{(E+i \Gamma)^{2}-\Delta^{2}}}
$$

When the structure is in the normal state, Eq. (15) reduces to the well-known expression $\dot{Q}_{e \text {-ph }}^{i, N}=\Sigma_{i} \mathcal{V}_{i}\left(T_{e}^{5}-T_{\text {bath }}^{5}\right)$ [3]. In our case, we approximate the normal-wire part of $\dot{Q}_{e \text {-ph }}^{d}$ with its normal-state value. As for $\alpha=3$, the minigap in the detector is almost suppressed. This choice leads to an underestimation of the SNR. We estimate numerically that the inverse proximity effect in the superconducting regions is not important given this approximation. 
The electron-phonon thermal conductances $G_{e \text {-ph }}^{N, S}$ can be obtained by differentiating Eq. (15) vs $T_{e}$ :

$$
\begin{aligned}
G_{\mathrm{th}}^{i}(T) & =\left.\frac{\partial \dot{Q}_{e-\mathrm{ph}}^{i}}{\partial T_{e}}\right|_{T_{e}=T_{\text {bath }}=T}=5 \Sigma_{i} \mathcal{V}_{i} T^{4} g_{i}(T) \\
g_{i}(T) & =\frac{1}{960 \zeta(5)} \int_{-\infty}^{\infty} \frac{d E d \varepsilon E|\varepsilon|^{3} M_{E, E-\varepsilon}^{i}}{k_{B}^{6} T^{6} \sinh \frac{\varepsilon}{2 k_{B} T} \cosh \frac{E}{2 k_{B} T} \cosh \frac{E-\varepsilon}{2 k_{B} T}} .
\end{aligned}
$$

Here, $g_{i}(T)$ is a dimensionless function obtaining the value $g_{i}(\infty)=1$ in the normal state or at high temperature and decreasing exponentially to $g(0)=(\Gamma / \Delta)^{2} \approx 0$ at low temperatures $k_{B} T \lesssim \Delta$ in the superconducting state.

Integration of Eq. (14) yields the electron-phonon relaxation halftime,

$\tau_{1 / 2}\left(\nu, T_{\text {bath }}\right)=\int_{\left[T_{e}^{\max }(\nu)+T_{\text {bath }}\right] / 2}^{T_{e}^{\max }(\nu)} d T_{e} \frac{C_{\text {tot }}^{e}\left(T_{e}\right)}{\dot{Q}_{e-\mathrm{ph}}^{\text {tot }}\left(T_{e}, T_{\text {bath }}\right)}$,

which allows us to determine the relevant thermal time constant of the detector for any given energy of the incoming photon. In Eq. (18), $T_{e}^{\max }(\nu)$ is the maximum electron temperature reached in the weak link after absorption of a photon of energy $h \nu$. For small temperature changes, the heat current can be linearized and written in terms of a thermal conductance $\dot{Q}^{\text {tot }}\left(T_{e}, T_{\text {bath }}\right) \simeq$ $\left(T_{e}-T_{\text {bath }}\right) G_{\text {th }}^{\text {tot }}\left(T_{\text {bath }}\right), \quad$ and, in this case, $\tau_{1 / 2} \simeq$ $\ln (2) C_{e}^{\text {tot }}\left(T_{\text {bath }}\right) / G_{\text {th }}^{\text {tot }}\left(T_{\text {bath }}\right)=\ln (2) \tau\left(T_{\text {bath }}\right) \quad$ is directly related to the linear thermal relaxation time $\tau\left(T_{\text {bath }}\right)$.

Finally, in the pulsed-mode operation, an important figure of merit is represented by the resolving power $h \nu / \delta E$ ( $\delta E$ is the energy resolution of full width at half maximum) [3]

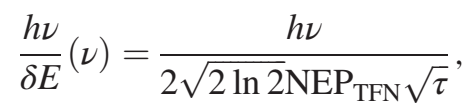

where $\mathrm{NEP}_{\mathrm{TFN}}$ is the thermal-fluctuation noise-limited noise equivalent power of the sensor which stems from thermal fluctuations between the electron and the lattice phonon system in the detector region. The resolving power is calculated for $T_{e}=T_{\text {bath }}$, i.e., in the idle state of the detector in the absence of radiation. In this case, the fluctuation-dissipation theorem states that at equilibrium, $\mathrm{NEP}_{\mathrm{TFN}}$ is given by [3]

$$
\mathrm{NEP}_{\mathrm{TFN}}=\sqrt{4 k_{B} T_{\mathrm{bath}}^{2} G_{\mathrm{th}}^{\mathrm{tot}}},
$$

where $G_{\mathrm{th}}^{\mathrm{tot}}=\partial \dot{Q}_{e \text {-ph }}^{\mathrm{tot}} / \partial T_{e}$ is the total thermal conductance of the detector. As a result, recalling that $C_{\mathrm{tot}}^{e}\left(T_{\mathrm{bath}}\right)=G_{\mathrm{th}}^{\mathrm{tot}} \tau$, the resolving power can also be written as

$$
\frac{h \nu}{\delta E}\left(\nu, T_{\text {bath }}\right)=\frac{h \nu}{4 \sqrt{2 \ln 2 k_{B} T_{\text {bath }}^{2} C_{\text {tot }}^{e}\left(T_{\text {bath }}\right)}},
$$

depending only on the electronic heat capacity.

The thermal energy fluctuations in the detector also contribute to the current noise measured in the readout

$$
\mathcal{S}_{I, \mathrm{tot}}=\mathcal{S}_{I, \mathrm{tun}}+\mathcal{S}_{I, \mathrm{TFN}},
$$

$$
\begin{aligned}
\mathcal{S}_{I, \mathrm{TFN}} & \simeq\left|\frac{d I_{p}}{d(h \nu)}\right|^{2} \frac{\mathrm{NEP}_{\mathrm{TFN}}^{2}}{\tau^{-2}+\omega^{2}} \\
& \simeq \frac{\left|I_{p}\left(0, T_{\mathrm{bath}}\right)-I_{p}\left[0, T_{e}(\delta E)\right]\right|^{2}}{\omega},
\end{aligned}
$$

where $\delta E=\mathrm{NEP}_{\mathrm{TFN}} / \sqrt{\omega}$ is the root-mean-square $(\mathrm{rms})$ energy fluctuation during time $t=2 / \omega$ for $\omega \gtrsim 1 / \tau$. The intrinsic noise $\mathcal{S}_{I, \text { tun }}$ of the tunneling is given by Eq. (8).

Figure 8 (a) shows the detector time constant $\tau_{1 / 2}$ calculated from Eq. (18) vs frequency $\nu$ for several values
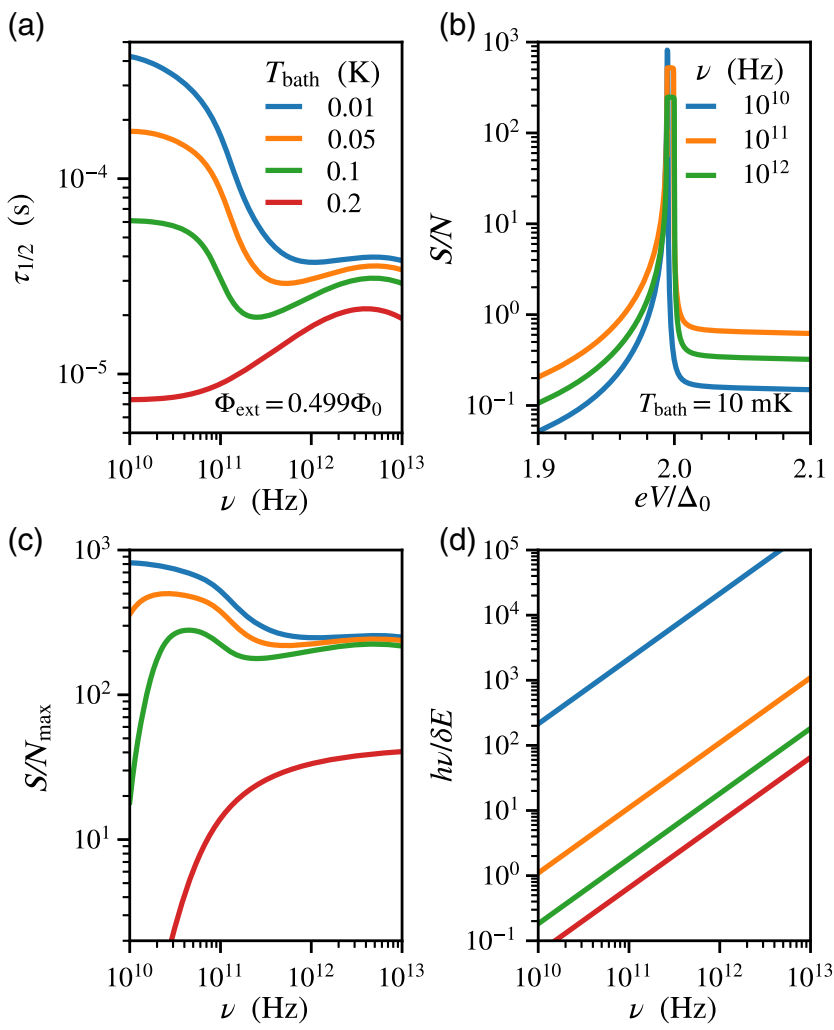

FIG. 8. (a) Detector time constant $\tau_{1 / 2}$ vs absorbed energy $h \nu$ of the incoming radiation calculated at different bath temperatures $T_{\text {bath. }}$ (b) Detector signal-to-noise ratio $(S / N)$ vs voltage $V$ calculated at $10 \mathrm{mK}$ for a few values of frequency of the incoming radiation. (c) Maximum $\operatorname{SNR}\left(S / N_{\max }\right)$ vs $\nu$ calculated for the same bath temperatures as in panel (a). (d) Detector resolving power $h \nu / \delta E$ vs $\nu$ calculated for the same $T_{\text {bath }}$ as in panel (a). In the calculations of panels (a)-(c), we set $\boldsymbol{\Phi}_{\text {ext }}=$ $0.499 \Phi_{0}$ and $\alpha=3$. 
of $T_{\text {bath }}$ at $\boldsymbol{\Phi}_{\text {ext }}=0.499 \boldsymbol{\Phi}_{0}$. From the figure, it turns out that $\tau_{1 / 2} \sim 10^{-5}-10^{-4} \mathrm{~s}$ in the range $T_{\text {bath }}=10-100 \mathrm{mK}$.

The detector signal-to-noise ratio $S / N$ at $10 \mathrm{mK}$ vs bias voltage $V$ across the SQUIPT is displayed in Fig. 8(b) for a few values of photon energy. Here, we set $\boldsymbol{\Phi}_{\text {ext }}=0.499 \boldsymbol{\Phi}_{0}$ and assume the measurement bandwidth $\omega=2 \pi / \tau_{1 / 2}$. We note, in particular, that the SNR is maximized close to $2 \Delta_{0} / e$ (compare to Fig. 4) and, depending on $V$, it obtains values $\gtrsim 500$. The maximum achievable SNR $\left(S / N_{\max }\right)$ vs photon frequency $\nu$ is shown in Fig. 8(c) for the same bath temperatures as in Fig. 8(a). The SNR is a nonmonotonic function of frequency, generally decreasing at high frequency and at low frequencies where $h \nu / \delta E<1$. Notably, SNRs of the order of 100 can be obtained in the whole frequency range for bath temperatures below $100 \mathrm{mK}$. Increasing $T_{\text {bath }}$ leads to a general reduction of the SNR.

The resolving power $h \nu / \delta E$ vs photon frequency calculated for the same $T_{\text {bath }}$ as in Fig. 8(a) is displayed in Fig. 8(d). In particular, the figure shows that resolving power values $>100$ can be achieved above $10 \mathrm{GHz}$ at $10 \mathrm{mK}$. At $100 \mathrm{mK}$, these values are significantly reduced due to the rapid increase in thermal fluctuations. The resolving power makes the detector of potential use in microwave and far-infrared single-photon detection.

The role of flux biasing of the interferometer is displayed in Fig. 9(a) where the SNR vs frequency at $10 \mathrm{mK}$ is shown for selected values of $\boldsymbol{\Phi}_{\text {ext }}$. In particular, moving away from $\boldsymbol{\Phi}=0.5 \boldsymbol{\Phi}_{0}$ leads to a reduction of the SNR in the lowfrequency end, and only significant deviations lead to suppression in the whole range.

The impact of the Dynes parameter $\Gamma$ on the signal-tonoise ratio is shown in Fig. 9(b), where $S / N_{\max }$ is calculated vs $\nu$ at $10 \mathrm{mK}$ for a few values of $\Gamma$. The result is computed using the same value in the detector and readout junctions and in the readout probe. In particular, sufficiently small values of $\Gamma$ have no effect on $S / N_{\max }$. The resolving power is similarly insensitive to it (not shown). The effect of $\Gamma$ mainly comes from the readout
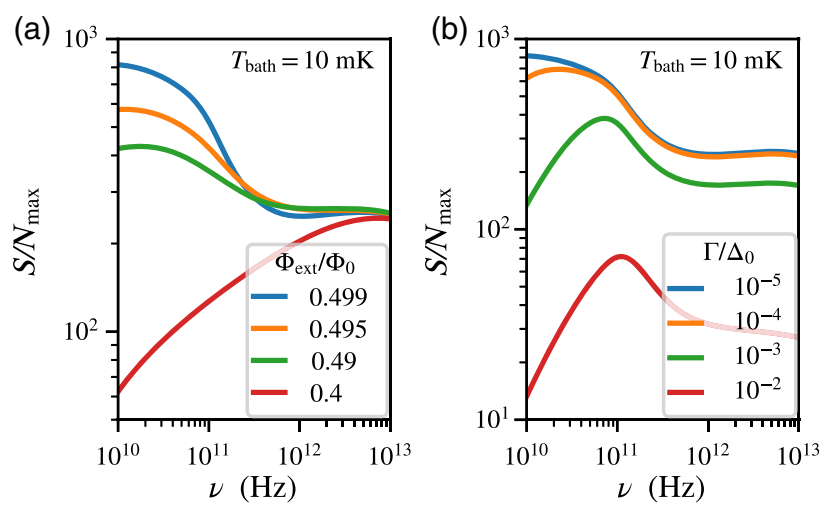

FIG. 9. (a) Detector maximum signal-to-noise ratio $S / N_{\max }$ vs frequency $\nu$ calculated for several values of $\boldsymbol{\Phi}_{\text {ext }}$. (b) $S / N_{\max }$ vs $\nu$ calculated for a few values of $\Gamma$ at $\boldsymbol{\Phi}_{\text {ext }}=0.499 \boldsymbol{\Phi}_{0}$. In all the calculations, we set $T_{\text {bath }}=10 \mathrm{mK}$. junction because under the operating conditions in the detector junction $\varphi^{d} \approx \pi$ so that the energy gap in the detector is $\varepsilon_{g}^{d} \ll \Delta$, and there are low-energy states contributing to heat capacity also at $\Gamma=0$.

In the above analysis, we assume the readout junction voltage bias does not fluctuate. A deviation from this limit results to photoassisted tunneling due to the fluctuations, and the convolution [37] with the noise can suppress the $S / N$ peak. To give an estimate of the effect, the fluctuations should be compared to the width of the maximum of $S / N$, which in Fig. 8(b) is $\left|\mathrm{eV}-2 \Delta_{0}\right| \lesssim 10^{-2} \Delta_{0}$. Modeling the noise from the measurement circuit with a $R-C$ circuit with resistance $R_{\text {env }} \ll R_{p}$ and parallel capacitance $C$, we have $\delta V_{\text {rms }} \simeq \sqrt{\pi \omega_{\text {env }} R_{\text {env }}^{2} \mathcal{S}_{I}}$, where $\omega_{\text {env }}=1 /\left(R_{\text {env }} C\right)$ sets the maximum detector bandwidth. For thermal noise $\mathcal{S}_{I}$ from room-temperature resistance $R_{\text {env }}, e \delta V_{\text {rms }} / \Delta_{0} \sim$ $0.006\left[R_{\text {env }} /(100 \Omega)\right]^{1 / 2}\left[\omega_{\text {env }} /\left(2 \pi \times 10^{5} \mathrm{~Hz}\right)\right]^{1 / 2}$. The junction shot noise can be neglected compared to the environment noise in this case. The effect of fluctuations can also be compared to the dependence on $\Gamma$ shown in Fig. 9 [38]. To estimate undesired signal cross-coupling from the antenna, consider a large input power $P_{\mathrm{opt}}=$ $10^{-15} \mathrm{~W}=10^{13} \mathrm{~Hz} \times h /\left(10^{-5} \mathrm{~s}\right.$ ) (c.f. the bolometer discussion in Sec. IV) dissipated in the readout junction: the corresponding fluctuation across the readout $S-N-S$ junction is $e \delta V_{\mathrm{rms}, r}=\sqrt{R_{r} P_{\mathrm{opt}}} \lesssim 0.001 \Delta_{0}$, a part of which can contribute to the voltage fluctuations over the tunnel probe. We expect that with sufficient filtering, voltage stability better than $e \delta V_{\mathrm{rms}} \sim 10^{-2} \Delta_{0}$ can then be achieved with standard room-temperature electronics, which is sufficient for the detector operation.

\section{NANOBOLOMETER}

\section{A. Thermal model}

The sensor operation in continuous power excitation (i.e., operation as a bolometer) can be described by considering those mechanisms that transport energy in the $N$ and $S$ parts of the detector. At low temperature, i.e., typically below $1 \mathrm{~K}$, the main contribution stems from electron-phonon heat flux which can be modeled according to Eq. (15). In particular, the incoming radiation is first absorbed by electrons in the weak link, while the lateral contacts with large superconducting gaps $\left(\Delta_{1}\right)$ prevent energy from escaping from the island. Then, the system can relax by releasing energy from electrons to the lattice phonons residing at $T_{\text {bath }}$. Under absorption of a continuous power $P_{\mathrm{opt}}$, the steady-state temperature $T_{e}$ in the weak link is determined for any $T_{\text {bath }}$ from the solution of the energy balance equation for incoming and outgoing power:

$$
P_{\mathrm{opt}}+\dot{Q}_{e-\mathrm{ph}}^{\mathrm{tot}}\left(T_{e}, T_{\mathrm{bath}}\right)=0 .
$$


Thanks to the reduced amplitude of $\dot{Q}_{e-\mathrm{ph}}^{\text {tot }}\left(T_{e}, T_{\text {bath }}\right)$ (and of the corresponding thermal conductance $G_{\text {th }}^{\text {tot }}$ ) at sufficiently low temperatures, Eq. (24) predicts that a fairly large electronic temperature can be established in the sensor even for a quite moderate absorbed optical power. It is instructive to show, first of all, the behavior of $G_{\text {th }}^{S} \approx G_{\text {th }}^{\text {tot }}$ obtained from Eq. (17) as a function of $T_{\text {bath. }}$. The sensor thermal conductance is displayed in Fig. 10(a) (dash-dotted line). In particular, $G_{\text {th }}^{\text {tot }}$ turns out to be somewhat suppressed with respect to that in the normal state $\left(G_{\text {th-N }}^{d}\right)$, and is reduced by an order of magnitude for $T_{\text {bath }} \lesssim 0.2 T_{c}$. These results for suppressed thermal conductance indicate that reduced $\mathrm{NEP}_{\mathrm{TFN}}$ (20) values can be achieved with a TPC-based nanobolometer.
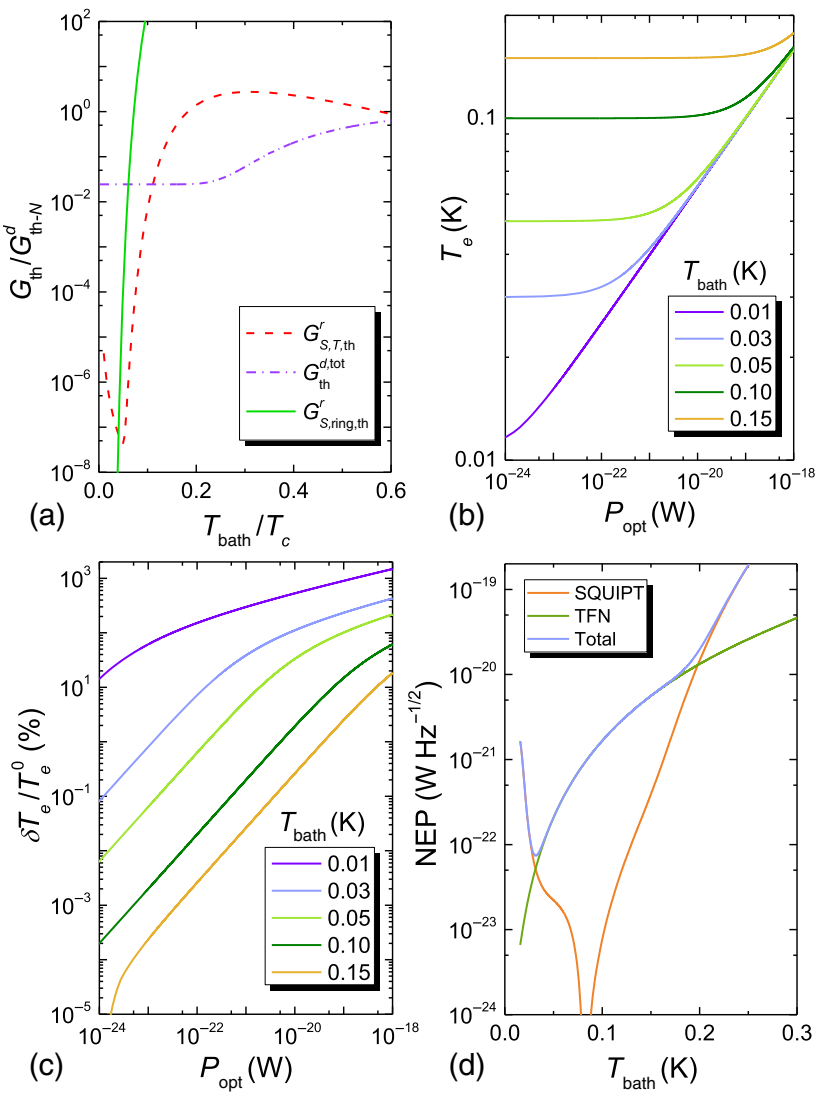

FIG. 10. (a) Weak-link total electron-phonon thermal conductance $G_{\text {th }}^{d \text {,tot }}$ of the detector vs $T_{\text {bath. }} \cdot G_{\text {th- } N}^{d}$ denotes the total $e$-ph thermal conductance in the normal state. The electronic heat conductivity of the SQUIPT $S$ tunnel probe and ring $\left(\Gamma / \Delta_{0}=10^{-5}, G_{N \text {,ring }}^{r}=10 \Omega^{-1}\right)$ are also shown for comparison. (b) Electronic temperature $T_{e}$ in the detector junction vs optical power $P_{\text {opt }}$ calculated for several values of $T_{\text {bath }}$. (c) Relative variation of the electronic temperature $\delta T_{e} / T_{e}^{0}$ vs $P_{\mathrm{opt}}$ calculated for the same bath temperatures as in panel (c). (d) NEP vs $T_{\text {bath }}$ for thermal-fluctuation noise (TFN) and shot noise (SQUIPT) at $V=1.995 \Delta_{0}$. In the calculations of panels (b)-(d), we set $\boldsymbol{\Phi}_{\mathrm{ext}}=0.499 \boldsymbol{\Phi}_{0}$ and $\alpha=3$.
As anticipated above, owing to the suppression of $G_{\text {th }}^{\text {tot }}$, a small absorbed optical power can markedly overheat electrons in the weak link, as shown in Fig. 10(b). In particular, at $T_{\text {bath }}=10 \mathrm{mK}$, power of $10^{-22} \mathrm{~W}$ can enhance $T_{e}$ up to $25 \mathrm{mK}$. At a higher bath temperature, the effect is less pronounced due to enhanced electronphonon interaction. For instance, at $50 \mathrm{mK}, T_{e}$ reaches $100 \mathrm{mK}$ for an input power of approximately $10^{-19} \mathrm{~W}$.

The relative variation of temperature $\delta T_{e} / T_{e}^{0}=$ $\left[T_{e}\left(P_{\mathrm{opt}}\right)-T_{e}\left(P_{\mathrm{opt}}=0\right)\right] / T_{e}\left(P_{\mathrm{opt}}=0\right)$ versus $P_{\mathrm{opt}}$ is displayed in Fig. 10(c) for the same values of $T_{\text {bath }}$ as in Fig. 10(b). In the bolometric configuration, $\delta T_{e} / T_{e}^{0}$ of the order of $10 \%$ can be obtained for $10^{-24} \mathrm{~W}$ and up to $300 \%$ for $10^{-20} \mathrm{~W}$ at $10 \mathrm{mK}$. At $T_{\text {bath }}=50 \mathrm{mK}, \delta T_{e} / T_{e}^{0}$ obtains values of order $0.01 \%$ and approximately $30 \%$ for the same $P_{\text {opt }}$.

We can also now comment on the assumption made above that the readout junction remains at temperature $T \approx T_{\text {bath }}$. The cooling of the readout weak link is provided by electronic heat transport to the $S$ tunnel probe and outdiffusion to the ring. The estimates for these can be written as $[3,39]$

$$
\begin{aligned}
G_{S, T, \text { th }}^{r} & =\frac{1}{4 e^{2} R_{p} k_{B} T^{2}} \int_{-\infty}^{\infty} d E \frac{E^{2} \mathcal{N}_{N}^{r}(E) \mathcal{N}_{S}^{r}(E)}{\cosh ^{2} \frac{E}{2 k_{B} T}}, \\
G_{S, \text { ring,th }}^{r} & \simeq G_{N, \text { ring,th }}^{r}(T) \int_{\Delta / k_{B} T}^{\infty} d x \frac{3 x^{2}}{2 \pi^{2} \cosh ^{2} \frac{x}{2}},
\end{aligned}
$$

where $\mathcal{N}$ are the densities of states on the two sides. The factor $G_{N, \text { ring,th }}^{r}(T)=L_{0} G_{N, \text { ring }}^{r} T$ is the normal-state electronic heat conductance from the junction to the thermal baths separated by the ring, where $L_{0}=\pi^{2} k_{B}^{2} /\left(3 e^{2}\right)$ is the Lorenz number, and $G_{N, \text { ring }}^{r}$ is the characteristic normalstate conductance between the detector and thermal baths. In order for the readout to remain close to $T_{\text {bath }}$ on the relevant detector timescales, we should have $G_{S \text {,th }}^{r}=$ $G_{S, T \text {,th }}^{r}+G_{S, \text { ring,th }}^{r} \gg G_{\text {th }}^{d \text {,tot }}$; i.e., this conductivity should be large compared to the electron-phonon heat conductivity of the detector junction. The above conductivities are shown in Fig. 10(a), together with $G_{\text {th }}^{d \text {,tot }}$, for the parameter choices assumed above. We can observe that it remains large compared to the electron-phonon heat conductivity at $T_{\text {bath }} \gtrsim 50 \mathrm{mK}$.

Finally, we wish to comment on the thermal transport out of the detector junction via the electron-photon heat conductance, $G_{e-\gamma}=\kappa(T) G_{Q}(T)$, where $G_{Q}=$ $\pi k_{B}^{2} T /(6 \hbar)=G_{Q, 0} T \approx 10^{-12} \mathrm{~W} / \mathrm{K}^{2} \times T$ is the thermal conductance quantum, and $\kappa(T)$ an impedance matching factor [40-42]. The matching factor $\kappa$ for BCS superconductors was considered in Ref. [43]. We can estimate $\kappa \sim 4 \operatorname{Re}\left[Z_{d}^{-1}\right] \operatorname{Re}\left[Z_{\text {env }}^{-1}\right] /\left|Z_{d}^{-1}+Z_{\text {env }}^{-1}\right|^{2}$, where $Z_{d}$ is the impedance of the detector and $Z_{\text {env }}$ that of its total 
electromagnetic environment, at thermal frequencies $\omega \lesssim$ $k_{B} T / \hbar$. For the detector junction at $k_{B} T \sim \hbar \omega \ll \Delta, Z_{d}^{-1} \simeq$ $G_{N}[(4 \Delta) /(\hbar \omega)] \sinh \left[(\hbar \omega) /\left(2 k_{B} T\right)\right] K_{0}\left[(\hbar \omega) /\left(2 k_{B} T\right)\right] e^{-\Delta /\left(k_{B} T\right)}-$ $i G_{N} \pi \Delta /(\hbar \omega)$ [22], where $K_{0}$ is a Bessel function so that $\kappa(T)=\kappa_{N}(T) \tilde{g}(T), \tilde{g}(T) \propto e^{-\Delta /\left(k_{B} T\right)}$. In the normal state, Ref. [42] obtained $\kappa_{N} \sim 10^{-3}$ for a mismatched circuit at $100 \mathrm{mK}$, which, in our case, corresponds [cf. Eq. (17)] to a crossover from dominant $e$-ph to dominant $e-\gamma$ at the temperature $T_{*}=\left[G_{Q, 0} \kappa_{N} /\left(5 \Sigma_{d} \mathcal{V}_{d}\right)\right]^{1 / 3} \times\left[\tilde{g}\left(T_{*}\right) / g(T)\right]^{1 / 3} \approx$ $50 \mathrm{mK} \times\left[\tilde{g}\left(T_{*}\right) / g\left(T_{*}\right)\right]^{1 / 3}$, and $g \sim \tilde{g}$ as both scale exponentially at $k_{B} T \ll \Delta$ due to the superconducting gap in the detector DOS. Note that the contribution of the superconducting elements and the SQUIPT readout to $\operatorname{Re}\left[Z_{\text {env }}^{-1}\right]$ are similarly suppressed, so that optimizing $G_{e-\gamma}$ is likely a problem of eliminating spurious couplings in the experimental setup.

\section{B. Performance: Noise equivalent power}

We now turn to discussing the achievable performance of the detector in the bolometric operation. In this configuration, an important figure of merit is represented by the noise equivalent power which stems from several uncorrelated sources of noise. In the present setup, the dominant contribution is due to thermal-fluctuation noise-limited noise equivalent power $\left[\mathrm{NEP}_{\mathrm{TFN}}\right.$; see Eq. (20)], whereas Johnson noise is absent owing to the operation of the Josephson junction in the dissipationless (i.e., supercurrent) regime. The $\mathrm{NEP}_{\mathrm{TFN}}$ is essentially independent of the Dynes parameter $\Gamma$. The contribution of the SQUIPT readout to NEP ( $\left.\mathrm{NEP}_{\text {SQUIPT }}\right)$ is determined by $\mathrm{NEP}_{\text {SQUIPT }}=G_{\text {th }}^{d \text {,tot }} s_{T}$, where $s_{T}$ is the temperature sensitivity discussed in Sec. II E.

Figure 10(d) shows the NEP vs $T_{\text {bath }}$. The minimal total NEP is approximately $10^{-22} \mathrm{~W} / \sqrt{\mathrm{Hz}}$ at $T_{\text {bath }} \approx 50 \mathrm{mK}$, and is determined by the thermal-fluctuation noise in the typical operation regime.

\section{CONCLUSIONS}

Combining (i) temperature-to-phase conversion due to kinetic inductance changes and (ii) SQUIPT tunneling spectroscopy for detection of the variation of the phase difference provides a basis for a thermal superconducting radiation detector. On the temperature-sensing side, the sensitivity of the device is boosted by the strong dependence of the SQUIPT tunneling current on the phase difference (Fig. 4). Moreover, it is influenced by the effect of the detector junction kinetic inductance variation on the phase difference of the readout junction, which is enhanced around the half-flux tuning point $\boldsymbol{\Phi} \approx \boldsymbol{\Phi}_{0} / 2$ (Fig. 3). The thermal sensitivity of the device is amplified by the small heat capacity of the detector junction, both due to superconductivity (Fig. 7) and the small volume, and by the reduction of electronic heat out-diffusion by the Andreev reflections from the superconductors. In principle, these aims could also be achieved using longer $S-N-S$ junctions not strictly in the short-junction regime $\left(L \lesssim \xi_{0}\right)$. Such a choice implies a different trade-off between the heat capacity (determined by volume and resistance) of the junction, the DOS gap giving the linear-response absorption threshold, and the kinetic inductance. We expect lithographic fabrication of both short [44] and long [17] junction devices is feasible.

For thermometry, the temperature-to-phase conversion differs from, e.g., $N-I-S$ sensors [3] in that the measurement is nonlocal; i.e., the readout junction is separated from the detector junction, and there is little electronic thermal coupling between them. Instead, the coupling is electrical, provided by supercurrent flow. This structure is useful for improved sensitivity, as maintaining the SQUIPT readout at a low temperature with cooling fins (here, the $S$ tunnel junction) improves its performance, and this cannot not be done inside the detector junction. We predict temperature sensitivities of tens of $\mathrm{nK} / \sqrt{\mathrm{Hz}}$ in a temperature range tunable over $T=10 \mathrm{mK}$ to $1 \mathrm{~K}$ with the choice of the magnetic flux.

In the calorimetric mode, assuming that sufficiently fast measurement of the $N-I-S$ tunneling current is made, resolving power (Fig. 8) of $h \nu / \delta E \approx 1-100$ is found in the range $\nu \approx 10^{10}-10^{13} \mathrm{~Hz}$, provided a low bath temperature below $T_{\text {bath }} \lesssim 100 \mathrm{mK}$ to suppress thermal noise. The operation frequency range is at somewhat lower frequencies, and the resolving power is higher when compared to an inductively coupled weak-link detector [13]. Compared to previously suggested small-volume superconducting nano-HEBs [2] operating in a similar temperature range, these numbers constitute an improvement in sensitivity; compared to Ref. [4], $\delta E$ will be 1-2 orders of magnitude smaller while retaining a similar thermal time constant $\tau$.

For the bolometric response, considering noise from the SQUIPT readout and thermal fluctuations, we find the NEP mostly dominated by the thermal-fluctuation noise of the detector junction for the relevant operation temperatures. Because of the small volume and superconductivity of the detector junction, we find NEP $\lesssim 10^{-21} \mathrm{~W} / \sqrt{\mathrm{Hz}}$ below $100 \mathrm{mK}$. This predicted NEP is below those obtained in recent sensitive sub-kelvin superconducting bolometers and kinetic inductance detectors, for which NEP $\gtrsim$ $10^{-20} \mathrm{~W} / \sqrt{\mathrm{Hz}}$ in similar millikelvin temperatures have been reported [2]. In comparison to the superconducting bolometers of Ref. [4], the difference is largely due to the smaller device volume and heat conductivity, as the limitation is due to intrinsic thermal-fluctuation noise.

In summary, we theoretically analyze a design and a readout scheme for a superconducting weak-link radiation detector. The performance numbers indicate an improvement over previously proposed weak-link detectors and will be competitive with other types of superconducting sensors operating in similar frequency and temperature 
ranges. We expect the design can be realized with current nanofabrication technology. The detector concept can be useful for the investigation of current open problems in astrophysics $[2,45]$ and quantum electronic circuits.

\section{ACKNOWLEDGMENTS}

F. G. and P. V. acknowledge the Ministero dell'Istruzione dell'Università e della Ricerca, Fondo per gli Investimenti della Ricerca di Base (MIUR-FIRB) Project Coca (Grant No. RBFR1379UX) and the European Research Council under the European Union's Seventh Framework Programme (FP7/2007-2013)/ERC Grant No. 615187COMANCHE for partial financial support.

[1] A. D. Semenov, G. N. Gol'tsman, and R. Sobolewski, Hotelectron effect in superconductors and its applications for radiation sensors, Supercond. Sci. Technol. 15, R1 (2002).

[2] B. S. Karasik, A. V. Sergeev, and D. E. Prober, Nanobolometers for $\mathrm{THz}$ photon detection, IEEE Trans. Terahertz Sci. Technol. 1, 97 (2011).

[3] F. Giazotto, T. T. Heikkilä, A. Luukanen, A. Savin, and J. Pekola, Opportunities for mesoscopics in thermometry and refrigeration: Physics and applications, Rev. Mod. Phys. 78, 217 (2006).

[4] J. Wei, D. Olaya, B. S. Karasik, S. V. Pereverzev, A. V. Sergeev, and M. E. Gershenson, Ultrasensitive hot-electron nanobolometers for terahertz astrophysics, Nat. Nanotechnol. 3, 496 (2008).

[5] B. Cabrera, R. M. Clarke, P. Colling, A. J. Miller, S. Nam, and R. W. Romani, Detection of single infrared, optical, and ultraviolet photons using superconducting transition edge sensors, Appl. Phys. Lett. 73, 735 (1998).

[6] B. S. Karasik, S. V. Pereverzev, A. Soibel, D. F. Santavicca, D. E. Prober, D. Olaya, and M. E. Gershenson, Energyresolved detection of single infrared photons with $\lambda=8 \mu \mathrm{m}$ using a superconducting microbolometer, Appl. Phys. Lett. 101, 052601 (2012).

[7] A. J. Kerman, E. A. Dauler, W. E. Keicher, J. K. W. Yang, K. K. Berggren, G. Gol'tsman, and B. Voronov, Kineticinductance-limited reset time of superconducting nanowire photon counters, Appl. Phys. Lett. 88, 111116 (2006).

[8] J. Govenius, R. E. Lake, K. Y. Tan, and M. Möttönen, Detection of Zeptojoule Microwave Pulses Using Electrothermal Feedback in Proximity-Induced Josephson Junctions, Phys. Rev. Lett. 117, 030802 (2016).

[9] D. F. Santavicca, B. Reulet, B. S. Karasik, S. V. Pereverzev, D. Olaya, M. E. Gershenson, L. Frunzio, and D. E. Prober, Energy resolution of terahertz single-photon-sensitive bolometric detectors, Appl. Phys. Lett. 96, 083505 (2010).

[10] N. Bluzer and M. G. Forrester, Superconducting quantum detectors, Opt. Eng. (Bellingham, Wash.) 33, 697 (1994).

[11] P. K. Day, H. G. LeDuc, B. A. Mazin, A. Vayonakis, and J. Zmuidzinas, A broadband superconducting detector suitable for use in large arrays, Nature (London) 425, 817 (2003).

[12] B. A. Mazin, Microwave kinetic inductance detectors: The first decade, AIP Conf. Proc. 1185, 135 (2009).
[13] F. Giazotto, T. T. Heikkilä, G. P. Pepe, P. Helistö, A. Luukanen, and J.P. Pekola, Ultrasensitive proximity Josephson sensor with kinetic inductance readout, Appl. Phys. Lett. 92, 162507 (2008).

[14] J. Govenius, R. E. Lake, K. Y. Tan, V. Pietilä, J. K. Julin, I. J. Maasilta, P. Virtanen, and M. Möttönen, Microwave nanobolometer based on proximity Josephson junctions, Phys. Rev. B 90, 064505 (2014).

[15] J. Voutilainen, M. A. Laakso, and T. T. Heikkilä, Physics of proximity Josephson sensor, J. Appl. Phys. 107, 064508 (2010).

[16] N. Bluzer, Analysis of quantum superconducting kinetic inductance photodetectors, J. Appl. Phys. 78, 7340 (1995).

[17] F. Giazotto, J. T. Peltonen, M. Meschke, and J. P. Pekola, Superconducting quantum interference proximity transistor, Nat. Phys. 6, 254 (2010).

[18] S. Gasparinetti, K. L. Viisanen, O. P. Saira, T. Faivre, M. Arzeo, M. Meschke, and J. P. Pekola, Fast Electron Thermometry for Ultrasensitive Calorimetric Detection, Phys. Rev. Applied 3, 014007 (2015).

[19] O. P. Saira, M. Zgirski, K. L. Viisanen, D. S. Golubev, and J.P. Pekola, Dispersive Thermometry with a Josephson Junction Coupled to a Resonator, Phys. Rev. Applied 6, 024005 (2016).

[20] M. Nahum and J. M. Martinis, Ultrasensitive hot-electron microbolometer, Appl. Phys. Lett. 63, 3075 (1993).

[21] F. Kos, S. E. Nigg, and L. I. Glazman, Frequency-dependent admittance of a short superconducting weak link, Phys. Rev. B 87, 174521 (2013).

[22] A. A. Abrikosov, L. P. Gor'kov, and I. M. Khalatnikov, A superconductor in a high frequency field, Zh. Eksp. Teor. Fiz. 35, 265 (1959) [Sov. Phys. JETP 8, 182 (1959)].

[23] M. J. Martínez-Pérez, A. Fornieri, and F. Giazotto, Rectification of electronic heat current by a hybrid thermal diode, Nat. Nanotechnol. 10, 303 (2015).

[24] I. O. Kulik and A. N. Omel'yanchuk, Contribution to the microscopic theory of the Josephson effect in superconducting bridges, JETP Lett. 21, 96 (1975).

[25] T. T. Heikkilä, J. Särkkä, and F. K. Wilhelm, Supercurrentcarrying density of states in diffusive mesoscopic Josephson weak links, Phys. Rev. B 66, 184513 (2002).

[26] E. Strambini, S. D’Ambrosio, F. Vischi, F. S. Bergeret, Yu. V. Nazarov, and F. Giazotto, The $\omega$-SQUIPT as a tool to phase-engineer Josephson topological materials, Nat. Nanotechnol. 11, 1055 (2016).

[27] M. Meschke, J. T. Peltonen, J. P. Pekola, and F. Giazotto, Tunnel spectroscopy of a proximity Josephson junction, Phys. Rev. B 84, 214514 (2011).

[28] S. D'Ambrosio, M. Meissner, C. Blanc, A. Ronzani, and F. Giazotto, Normal metal tunnel junction-based superconducting quantum interference proximity transistor, Appl. Phys. Lett. 107, 113110 (2015).

[29] A. Ronzani, S. D’Ambrosio, P. Virtanen, F. Giazotto, and C. Altimiras, Phase-driven collapse of the Cooper condensate in a nanosized superconductor, Phys. Rev. B 96, 214517 (2017).

[30] S. N. Artemenko, A. F. Volkov, and A. V. Zaitsev, Theory of nonstationary Josephson effect in short superconducting contacts, Zh. Eksp. Teor. Fiz. 76, 1816 (1979) [Sov. Phys. JETP 49, 924 (1979)]. 
[31] F. Giazotto and F. Taddei, Hybrid superconducting quantum magnetometer, Phys. Rev. B 84, 214502 (2011).

[32] M. Tinkham, Introduction to Superconductivity, 2nd ed. (McGraw-Hill, New York, 1996).

[33] D. Rogovin and D. J. Scalapino, Fluctuation phenomena in tunnel junctions, Ann. Phys. (N.Y.) 86, 1 (1974).

[34] P. Virtanen, F. Vischi, E. Strambini, M. Carrega, and F. Giazotto, Quasiparticle entropy in superconductor/normal metal/superconductor proximity junctions in the diffusive limit, Phys. Rev. B 96, 245311 (2017).

[35] N. B. Kopnin, Theory of Nonequilibrium Superconductivity, International Series of Monographs on Physics, No. 110 (Oxford University Press, New York, 2001).

[36] A. V. Timofeev, C. P. García, N. B. Kopnin, A. M. Savin, M. Meschke, F. Giazotto, and J. P. Pekola, RecombinationLimited Energy Relaxation in a Bardeen-Cooper-Schrieffer Superconductor, Phys. Rev. Lett. 102, 017003 (2009).

[37] G. L. Ingold and Yu. V. Nazarov, Charge tunneling rates in ultrasmall junctions, in Single Charge Tunneling, NATO ASI Series B Vol. 294, edited by H. Grabert and M. H. Devoret (Plenum Press, New York, 1992), Chap. 2, pp. 21-107.

[38] J. P. Pekola, V. F. Maisi, S. Kafanov, N. Chekurov, A. Kemppinen, Yu. A. Pashkin, O.P. Saira, M. Möttönen, and J. S. Tsai, Environment-Assisted Tunneling as an Origin of the Dynes Density of States, Phys. Rev. Lett. 105, 026803 (2010).

[39] J. Bardeen, G. Rickayzen, and L. Tewordt, Theory of the thermal conductivity of superconductors, Phys. Rev. 113, 982 (1959).

[40] J. B. Pendry, Quantum limits to the flow of information and entropy, J. Phys. A 16, 2161 (1983).

[41] K. Schwab, E. A. Henriksen, J. M. Worlock, and M. L. Roukes, Measurement of the quantum of thermal conductance, Nature (London) 404, 974 (2000).

[42] A. V. Timofeev, M. Helle, M. Meschke, M. Möttönen, and J. P. Pekola, Electronic Refrigeration at the Quantum Limit, Phys. Rev. Lett. 102, 200801 (2009).

[43] R. Bosisio, P. Solinas, A. Braggio, and F. Giazotto, Photonic heat conduction in Josephson-coupled Bardeen-CooperSchrieffer superconductors, Phys. Rev. B 93, 144512 (2016).

[44] N. Ligato, G. Marchegiani, P. Virtanen, E. Strambini, and F. Giazotto, High operating temperature in $v$-based superconducting quantum interference proximity transistors, Sci. Rep. 7, 8810 (2017).

[45] L. M. Capparelli, G. Cavoto, J. Ferretti, F. Giazotto, A. D. Polosa, and P. Spagnolo, Axion-like particle searches with sub-THz photons, Phys. Dark Universe 12, 37 (2016). 